\title{
A Molecular Dynamics Approach to Calculate the Thermodiffusion (Soret and Seebeck) Coefficients of Salts in Aqueous Solutions
}

\author{
Leandro Rezende Franco, ${ }^{*}$ André Luiz Sehnem, ${ }^{*}$ Antônio Martins Figueiredo \\ Neto, ${ }^{*}$ and Kaline Coutinho* \\ Universidade de Sao Paulo, Instituto de Fisica, Cidade Universitaria, 05508-090 Sao \\ Paulo, SP, Brazil
}

E-mail: leofranc@if.usp.br; alsehnem@if.usp.br; afigueiredo@if.usp.br; kaline@if.usp.br

\begin{abstract}
An approach to investigate the physical parameters related to the ions thermodiffusion in aqueous solution is proposed herein by calculating the equilibrium hydration free energy and the self-diffusion coefficient as a function of temperature, ranging from 293 to $353 \mathrm{~K}$, using molecular dynamics simulations of infinitely diluted ions in aqueous solutions. Several ion force field parameters are used in the simulations and new parameters are proposed for some ions to better describe their hydration free energy. Such a theoretical framework enables the calculation of some single-ion properties, such as heat of transport, Soret coefficient and mass current density, as well as properties of salts, such as effective mass and thermal diffusion, Soret and Seebeck coefficients. These calculated properties are compared with experimental data available from optical measurements and showed good agreement revealing an excellent theoretical predictability of salt thermodiffusion properties. Differences in single-ion Soret and self-diffusion coefficients of anions and cations give rise to a thermoelectric field,
\end{abstract}


which affects the system response that is quantified by the Seebeck coefficient. The fast and slow Seebeck coefficients are calculated and discussed, resulting in values with $\mathrm{mV} / \mathrm{K}$ order-of-magnitude, as observed in experiments involving several salts, such as $\mathrm{K}^{+} \mathrm{Cl}^{-}, \mathrm{Na}^{+} \mathrm{Cl}^{-}, \mathrm{H}^{+} \mathrm{Cl}^{-}, \mathrm{Na}^{+} \mathrm{OH}^{-}, \mathrm{TMA}^{+} \mathrm{OH}^{-}$and $\mathrm{TB} A^{+} \mathrm{OH}^{-}$. The present approach can be adopted for any ion or charged particle dispersed in water with the aim of predicting the thermoelectric field induced through the fluid. It has potential applications in designing electrolytes for ionic thermoelectric devices in order to harvest energy and thermoelectricity in biological nanofluids. 


\section{Introduction}

The need to increase and diversify the world energy matrix has lead to the emergence of research on multiple energy sources, including low-grade harvesting devices with optimized thermoelectric response. ${ }^{1,2}$ In liquid electrolytes, or ionic liquids, there is a strong Seebeck effect, which the electrostatic potential drops induced by a temperature difference. ${ }^{1,3}$ The thermoelectric energy is enhanced increasing the electrical conductivity $\sigma$ and Seebeck coefficient $S$, and decreasing the thermal conductivity $\kappa$ of the fluid. ${ }^{4}$ Aqueous electrolytes have a higher figure of merit (for efficiency assessment) when compared to non-aqueous electrolytes, mainly due to their higher $\sigma$-values. ${ }^{5}$ Seebeck coefficient $S$ of electrolytes depends on the single-ion Soret coefficient $\alpha_{i}$, which is defined as the ratio of a concentration change of ions, or charged molecules or particles, induced by a temperature difference in aqueous solution, i.e. it depends on the tendency of spatial separation of negative and positive charge carries induced by the thermal gradient. Therefore, Soret effect describes ionic diffusive motion that originates from a temperature gradient along its direction, defining the thermodiffusion effect. ${ }^{6}$ It is different from self-diffusion, or tracer diffusion, which is a spontaneous random movement of particles (neutral or charged) in the absence of concentration (or chemical potential) gradient.

Single-ion Soret coefficient $\alpha_{i}$ is related to single-ion heat of transport or entropy of transport, ${ }^{7} Q_{i}^{*}$ and $S_{i}^{*}$ respectively, arising from the temperature dependence upon interactions between ions and solvent molecules. ${ }^{8}$ Knowledge on the underlying physical mechanisms, i.e. the nature of specific interactions that drive charge carriers in temperature gradients, leads to an optimization of low-grade energy devices based on aqueous electrolytes, such as thermoelectric supercapacitors and thermopiles. ${ }^{1,5,9}$ New electrolytes and ionic liquids are promising candidates to optimize such devices. However, even for the case of a monovalent and monoatomic salt dissociated in water, it is impossible to predict their performance at generating thermoelectric energy due to the absence of a thorough description of thermodiffusive effects. 
The presence of temperature gradients in aqueous salty solutions induces an increasing ion concentration in the cold or hot side of the solution. ${ }^{7,10,11}$ This is due to the thermodiffusion effect that generates an ionic flux which is generally given by the mass current density

$$
j_{T i}=-n_{i} \alpha_{i} D_{i} \nabla T / T,
$$

where $i=+$ for cations and $i=-$ for anions, $n_{i}$ and $D_{i}$ are the numerical volumetric concentrations and the self-diffusion coefficient of ion $i$, and $\nabla T$ is the temperature gradient. Accumulation of ions means that, in accordance with Fick law of diffusion, there is a counter ion-flux given by $j_{n i}=-D_{i} \nabla n_{i}$. Therefore, the single-ion Soret coefficient $\alpha_{i}$ characterizes ion thermodiffusion. Difference in $\alpha_{i}$ between the positive and negative ions have been assigned to generate charge accumulation. An electrostatic potential drop arises in the temperature gradient, for which the Seebeck coefficient is defined as the potential over the temperature differences. A first out-of-equilibrium thermodynamic description of the aforementioned effect was issued by Eastman nearly 100 years ago ${ }^{12,13}$ who had proposed a relation between $\alpha_{i}$ and entropy of transport $S_{i}^{*}$. Agar et al. have related the salt Soret coefficient that is experimentally accessible ${ }^{14,15}$ to the single-ion heat of transport $Q_{i}^{*}{ }^{8}$

In recent years, scientific interest focused on experimental measurements to reveal elementary aspects of the Soret effect. The nature of ions ${ }^{16-18}$ such as the hydrophilic/hydrophobic degree has an important role in driving solute migration towards the cold or hot side of the solution in temperature gradients. ${ }^{19,20}$ As regards electrolytes, differences in $\alpha_{i}{ }^{21}$ or $Q_{i}^{* 7}$ and in $D_{i}{ }^{22}$ of anions and cations are predicted to generate a thermoelectric field, which leads to thermophoresis of charged nanoparticles ${ }^{18,23-25}$ and it has been increasingly investigated so that it can be applied in energy harvesting. ${ }^{1,2,26,27}$

Some theoretical concepts of thermodiffusion in a given solution have been developed based on the temperature dependence of the particle free energy. ${ }^{28-30}$ They considered a simple model where the solution was depicted in many thin-slabs cells comparing to the 
volume of the total solution, but macroscopic comparing to the particle size. From the reference cell at temperature $T$, neighbor cells are at different temperatures $T-\delta T$ and $T+\delta T$ towards cold and hot directions, respectively, in the temperature gradient. The probability of the particle move to the neighboring cell is related to the change in entropy, thus causing absorption or heat release. This single-ion heat of transport $Q_{i}^{*}$ was defined as $Q_{i}^{*}=T\left(d G_{i} / d T\right)$, where $G_{i}$ is the Gibbs free energy of the single-ion/solvent system in local equilibrium. It is the basis for describing a non-equilibrium effect using equilibrium parameters. The single-ion Soret coefficient equation was defined as ${ }^{12,30}$

$$
\alpha_{i}=\frac{Q_{i}^{*}}{2 k_{B} T}=\frac{1}{2 k_{B}} \frac{d G_{i}}{d T}
$$

where positive single-ion Soret coefficient $\alpha_{i}$ indicates the ion movement to the cold side through the temperature gradient. This equation has been applied in experimental results with charged micro-particles by Duhr and Braun. ${ }^{28}$

Microscopic features of aqueous solutions that lead to the ionic Soret effect raises a fundamental question, i.e. whether the calculation of ion free energy is enough to describe the ionic Soret effect and whether interactions other than electrostatic ones also play a major role.

Takeyama and Nakashima ${ }^{31}$ showed proportionality between the single-ion heat of transport $Q_{i}^{*}$ and ion hydration entropy, $Q_{i}^{*}=-f_{i}^{T N} T S_{i}^{h y d}$, using experimental data. Therefore, as Equation 2 suggests, a starting point to describe the thermodiffusion of single ions in aqueous solution is to calculate the hydration free energy at different temperatures, $G_{i}^{\text {hyd }}(T)$, and obtain the ion hydration entropy as $-S_{i}^{h y d}=d G_{i}^{h y d} / d T$. Then, $G_{i}^{\text {hyd }}$ of ions can be calculated by molecular dynamics (MD) simulations at atomistic level at specific temperature conditions.

Carlsson and Aqvist ${ }^{32}$ used MD simulations combined with perturbation technique to calculate the hydration free energy $G_{i}^{h y d}$ for the monoatomic cations $\left(L i^{+}, N a^{+}, K^{+}, R b^{+}\right.$ and $\mathrm{Cs}^{+}$). Their results present a positive free energy variation by a temperature increase, 
$\Delta G_{i}^{\text {hyd }} / \Delta T>0$, in which values of hydration free energy at $298 \mathrm{~K}$ are in good agreement with experimental data. Recently Niether et al. ${ }^{33,34}$ have analyzed the connection between hydrophilicity/hydrophobicity and thermodiffusion of biomolecules in aqueous solution. These works suggest that the temperature dependence on the total interaction between ions and water molecules, which is calculated by $G_{i}^{h y d}(T)$, may be the most thorough physical description of the thermodiffusion of (molecular) ions and biomolecules. However, although there are some theoretical studies about the dependence of $G_{i}^{\text {hyd }}(T)$ on temperature in aqueous solution and how it is associated with thermodiffusion, it is found no calculation of thermodiffusionrelated coefficients, such as the Soret coefficient (Equation 2) by using $G_{i}^{\text {hyd }}(T)$ calculated through computational methods. Lecce et al. ${ }^{35,36}$ have performed recently computational simulations using non-equilibrium MD to calculate thermodiffusion coefficients of finite concentrations of $\mathrm{Li}^{+} \mathrm{Cl}^{-}$in aqueous solution. Their computational approach calculates the heat of transport for anions and cations in each simulation, which requires multiple ions and thousands of water molecules for each simulation and demands long computational time. They reveal substantial differences between transport coefficients at infinite dilution and finite concentrations.

Therefore, MD simulations of infinitely diluted aqueous solutions of ions have been used in this work in order to evaluate the dependence on hydration free energy on temperature, $\Delta G_{i}^{h y d} / \Delta T$, using the Bennett Acceptance Ratio (BAR) method. We performed simulations for different types of ions in water, including the monoatomic alkali halide ions $\left(\mathrm{Na}^{+}, \mathrm{K}^{+}\right.$and $C l^{-}$), the Tetra-n-alkyl-ammonium $(T A A)$ with $n=1$ (Tetramethylammonium, $T M A^{+}$) and 4 (Tetrabutylammonium, $T B A^{+}$), hydronium $H_{3} O^{+}$and hydroxide $O H^{-}$ions. We adopted two force field models for water and four force field parameters for monoatomic ions. For hydroxide and hydronium, we adopted available force field parameters, but we also propose a new set of non-bonded parameters for these ions in order to better reproduce their hydration free energies at room temperature. Moreover, we propose a new set of atomic charge distribution for $T A A$ ions within the force field parameters. Additionally, we 
calculated ionic self-diffusion coefficients at different temperatures, $D_{i}(T)$, using the slope of mean square displacement (MSD). This extensive investigation offers a complete overview of the temperature dependence on the hydration and mobility of ions that have been used as basis to understand the microscopic structure of ions and thermodiffusion of salts. In this context, some single-ion properties were calculated, such as the Soret coefficient $\alpha_{i}$, heat of transport coefficient $Q_{i}^{*}$ and mass current density $j_{T i}$; in addition to some salt properties, such as effective mass diffusion $D$, thermal diffusion coefficient $D_{T}$, Soret $S_{T}$ and Seebeck $S$ coefficients.

A thermodiffusive interdependence between anions and cations is associated with the salt concentration gradient and the thermoelectric field arising along a temperature gradient in ionic solution. An elementary source of ion thermodiffusion has been assumed according to different probabilities of moving towards the cold or hot side of the solution, ${ }^{29,37}$ depending on the difference in hydration free energy. An investigation of ions was carried out based on a comparison of calculated values with experimental data available for $G_{i}^{h y d}(T)$ and $\alpha_{i}$. From calculated $\alpha_{i}$ and $D_{i}$, we estimate the salt Soret $S_{T}$ and Seebeck $S$ coefficients of ionic solutions. The dependence of thermal diffusion coefficient $D_{T}$ on temperature, a broadly discussed topic in literature on thermodiffusion, shows a linear behavior with temperature as a consequence of the temperature independence of the single-ion Soret coefficient $\alpha_{i}$ and a linear dependence of effective mass diffusion $D(T)$ for all ions. For describing the thermoelectric effect, Seebeck coefficient was calculated using a recent theoretical expression, ${ }^{22}$ with agreements in the same orders of magnitude for monovalent salt in aqueous solutions. These analyses validate our procedure to computationally estimate thermodiffusion-related coefficients of charged molecules/particles in water and indicate the method to be used in the development of complex electrolytes for thermoelectric energy-harvesting devices. 


\section{Results and discussion}

The results are going to be presented in four sections. Firstly, those obtained from MD simulations are going to be shown in order to discuss their physical framework based on the hydration free energy dependence on temperature for assessing the thermodiffusion effect in electrolyte solutions. Values of the hydration free energies $G_{i}^{\text {hyd }}(T)$ for ions obtained using different force field parameters are going to be presented and compared with available experimental data. ${ }^{38-40}$ Next, ions self-diffusion coefficients $D_{i}(T)$ obtained from MD simulations for different temperatures will be presented, discussed and compared with experimental data. ${ }^{8,41}$ Additionally, the values calculated for salts effective mass diffusion $D(T)$ at different temperatures are shown, discussed and compared with experimental data. ${ }^{16,42}$ Then, the values calculated for single-ions properties: Soret coefficient $\alpha_{i}$, heat of transport coefficient $Q_{i}^{*}$ and mass current density $j_{T i}$ are shown. The values calculated for the singleion heat of transport $Q_{i}^{*}$ are compared with experimental values obtained by Agar et al., ${ }^{8}$ and those for the single-ion mass current density $j_{T i}$ are discussed by showing a classification scale of ion thermodiffusion at the same concentration $n_{i}$ and temperature gradient $\nabla T / T$ conditions. Lastly, the calculated values for salts thermodiffusion-related coefficients, i.e. thermal diffusion $D_{T}$, Soret $S_{T}$ and Seebeck at fast and slow time regimes, $S_{\text {fast }}$ and $S_{\text {slow }}$ respectively, are presented, discussed and compared with experimental values obtained from optical experiments. ${ }^{16,42,43}$

\subsection{Ion hydration}

The hydration free energy $G_{i}^{h y d}(T)$ for studied ions have been calculated at thirteen different

temperatures, in the range of 293 and $353 \mathrm{~K}$ in intervals of $5 \mathrm{~K}$. In Table 1 , the $G_{i}^{h y d}(T)$ values are shown only for five temperatures for simplification. We choose the four values equally spaced between the temperature interval (293, 313, 333 and $353 \mathrm{~K}$ ) and additionally the room temperature $(298 \mathrm{~K})$ to compare with the most abundant data in the literature. ${ }^{38-40}$ 
Different ionic force field parameters ${ }^{44-52}$ are identified in the second column of Table 1 and their parameters are shown in the Supporting Information (SI). In Table 1, only the results obtained for MD simulations using the SPC/E water model are presented. The results obtained using the TIP3P water model are presented in SI.

Table 1: Hydration Free Energies $G_{i}^{\text {hyd }}(T)$ (in kcal/mol) for ions in aqueous solution calculated using the BAR method in MD simulations at different temperatures with different force field parameters for ions and the SPC/E water model. The uncertainty of the last digit is in parenthesis and the experimental values at room temperature are in brackets.

\begin{tabular}{|c|c|c|c|c|c|c|c|}
\hline Ion & Force Field & $G_{i}^{h y d}(293)$ & $G_{i}^{h y d}$ & $(298)$ & $G_{i}^{h y d}(313)$ & $G_{i}^{h y d}(333)$ & $G_{i}^{h y d}(353)$ \\
\hline \multirow{4}{*}{$C l^{-}$} & Canongia $^{44}$ & $-104.2(1)$ & $-103.8(1)$ & \multirow{4}{*}{$\begin{array}{l}{[-89.1]^{38}} \\
{[-81.3]^{39}}\end{array}$} & $-103.5(1)$ & $-102.8(2)$ & $-102.0(2)$ \\
\hline & $\operatorname{Dang}^{45}$ & $-90.2(2)$ & $-90.1(1)$ & & $-89.7(1)$ & $-89.2(2)$ & $-88.8(1)$ \\
\hline & Jorgensen $^{46}$ & $-91.2(2)$ & $-91.0(1)$ & & $-90.7(1)$ & $-90.1(1)$ & $-89.8(2)$ \\
\hline & $\operatorname{Roux}^{47}$ & $-96.6(2)$ & $-96.1(2)$ & & $-95.8(1)$ & $-95.2(2)$ & $-94.7(1)$ \\
\hline \multirow{4}{*}{$K^{+}$} & Aqvist $^{48}$ & $-64.4(1)$ & $-64.4(2)$ & \multirow{4}{*}{$\begin{array}{l}{[-71.2]^{38}} \\
{[-70.5]^{39}}\end{array}$} & $-64.4(1)$ & $-64.1(1)$ & $-63.8(1)$ \\
\hline & $\operatorname{Dang}^{49}$ & $-64.9(1)$ & $-64.7(1)$ & & $-64.4(1)$ & $-64.4(2)$ & $-64.2(1)$ \\
\hline & Jorgensen ${ }^{46}$ & $-59.3(2)$ & $-59.3(1)$ & & $-59.0(0)$ & $-59.0(2)$ & $-58.8(1)$ \\
\hline & $\operatorname{Roux}^{50}$ & $-69.0(1)$ & $-68.9(1)$ & & $-68.8(1)$ & $-68.6(1)$ & $-68.5(1)$ \\
\hline \multirow{4}{*}{$N a^{+}$} & Aqvist $^{48}$ & $-82.3(2)$ & $-82.0(1)$ & \multirow{4}{*}{$\begin{array}{l}{[-88.7]^{38}} \\
{[-87.2]^{39}}\end{array}$} & $-81.9(1)$ & $-81.6(1)$ & $-81.2(1)$ \\
\hline & $\operatorname{Dang}^{45}$ & $-89.2(1)$ & $-89.0(1)$ & & $-88.9(1)$ & $-88.4(1)$ & $-87.9(1)$ \\
\hline & Jorgensen $^{46}$ & $-76.7(1)$ & $-76.5(1)$ & & $-76.2(1)$ & $-76.1(1)$ & $-75.8(1)$ \\
\hline & $\operatorname{Roux}^{50}$ & $-91.8(2)$ & $-91.6(1)$ & & $-91.3(1)$ & $-90.8(0)$ & $-90.2(1)$ \\
\hline \multirow{4}{*}{$O H^{-}$} & Netz $^{51}$ & $-124.4(1)$ & $-124.1(2)$ & \multirow{4}{*}[-105.0]{$^{40}$} & $-123.2(2)$ & $-122.6(1)$ & $-121.3(2)$ \\
\hline & Jorgensen $^{46}$ & $-130.0(3)$ & $-129.9(3)$ & & $-128.9(2)$ & $-127.6(1)$ & $-126.8(2)$ \\
\hline & $20-$ sites $^{52}$ & $-136.7(3)$ & $-136.3(3)$ & & $-135.9(1)$ & $-133.7(2)$ & $-133.0(2)$ \\
\hline & Ours & $-107.4(2)$ & $-107.2(3)$ & & $-106.7(1)$ & $-106.2(3)$ & $-105.4(3)$ \\
\hline \multirow{2}{*}{$\mathrm{H}_{3} \mathrm{O}^{+}$} & $\operatorname{Netz}^{51}$ & $-103.6(1)$ & $-103.4(2)$ & \multirow{2}{*}[-110.4]{$^{40}$} & $-102.8(1)$ & $-101.9(2)$ & $-101.1(2)$ \\
\hline & Ours & $-110.7(3)$ & $-110.5(1)$ & & $-109.6(3)$ & $-108.6(3)$ & $-108.0(3)$ \\
\hline$T M A^{+}$ & Ours & $-37.0(2)$ & $-36.9(2)$ & - & $-36.3(2)$ & $-35.7(1)$ & $-35.2(4)$ \\
\hline$T B A^{+}$ & Ours & $-26.3(4)$ & $-26.1(3)$ & - & $-24.6(2)$ & $-23.2(3)$ & $-22.1(3)$ \\
\hline
\end{tabular}

As expected, all $G_{i}^{h y d}(T)$ are negative and alkyl ions, $T B A^{+}$and $T M A^{+}$, are less hydrophilic than the others. The values calculated for $T B A^{+}$and $T M A^{+}$are $G_{i}^{h y d}(298)=$ $-26.1 \mathrm{kcal} / \mathrm{mol}$ and $-36.9 \mathrm{kcal} / \mathrm{mol}$, respectively. As for $K^{+}$, the values of $G_{i}^{\text {hyd }}(298)$ are from -59.3 to $-68.9 \mathrm{kcal} / \mathrm{mol}$, while experimental values are -70.5 and $-71.2 \mathrm{kcal} / \mathrm{mol} .{ }^{38,39}$ As 
regards $\mathrm{Na}^{+}$, values are ranging between -76.5 and $-91.6 \mathrm{kcal} / \mathrm{mol}$, but experimental values are -87.2 and $-88.7 \mathrm{kcal} / \mathrm{mol} .{ }^{38,39}$ With respect to $\mathrm{Cl}^{-}$, calculated values range between -90.1 and $-103.8 \mathrm{kcal} / \mathrm{mol}$, and experimental values are -81.3 and $-89.1 \mathrm{kcal} / \mathrm{mol} .{ }^{38,39}$ Note that there is a broad difference of around $8 \mathrm{kcal} / \mathrm{mol}$ in the two experimental data and all force field parameters for $\mathrm{Cl}^{-}$present $G_{i}^{\text {hyd }}(298)$ closer to the highest experimental value. Concerning $\mathrm{OH}^{-}$and $\mathrm{H}_{3} \mathrm{O}^{+}$, values are from -107.2 to $-136.3 \mathrm{kcal} / \mathrm{mol}$ and from -103.4 to $-110.5 \mathrm{kcal} / \mathrm{mol}$, and experimental values are -105.0 and $-110.4 \mathrm{kcal} / \mathrm{mol},{ }^{40}$ respectively. The differences between the experimental and theoretical values of the hydration free energy, $\Delta G_{i}^{\text {hyd }}$ (exp/theor), at $298 \mathrm{~K}$ are shown in Figure 1 considering different experimental values, ionic force field parameters and water models. It can be seen that the best agreement between experimental/theoretical values (less than $1.5 \mathrm{kcal} / \mathrm{mol}$ ) is observed for the ionic force field parameters proposed by: Smith and Dang ${ }^{45}$ for $\mathrm{Cl}^{-}$and $\mathrm{Na}^{+}$, Beglov and Roux ${ }^{50}$ for $\mathrm{K}^{+}$and us (those proposed herein) for $\mathrm{OH}^{-}$and $\mathrm{H}_{3} \mathrm{O}^{+}$. As a general trend, both water models present similar values for $G_{i}^{\text {hyd }}$ but with TIP3P model the $\Delta G_{i}^{\text {hyd }}$ (exp/theor) values are slightly smaller than with SPC/E.

For all anions and cations, $G_{i}^{\text {hyd }}(T)$ becomes increasingly negative as temperature becomes lower, i.e. $\Delta G_{i}^{\text {hyd }} / \Delta T>0$. The $G_{i}^{\text {hyd }}(T)$ behavior with respect to temperature is better depicted in Figure 2 for ionic force field parameters that achieved the best experimental/theoretical agreement and the SPC/E water model. Regardless of ion charge, the dependence of $G_{i}^{\text {hyd }}(T)$ on temperature is linear, and all of which are better hydrated at lower temperatures, but the angular coefficient of the best linear fit is force-field dependent. As a general trend, it was observed that polyatomic ions $\left(\mathrm{H}_{3} \mathrm{O}^{+}, \mathrm{OH}^{-}, \mathrm{TBA}^{+}\right.$and $\left.T M \mathrm{~A}^{+}\right)$ are more sensitive to temperature variation, i.e. $\Delta G_{i}^{h y d} / \Delta T$ is larger for polyatomic ions than for monoatomic ions $\left(\mathrm{Cl}^{-}, \mathrm{Na}^{+}\right.$and $\left.\mathrm{K}^{-}\right)$.

By calculating $G_{i}^{h y d}$, two contributions can be obtained separately, which are the electrostatic and non-electrostatic terms $G_{i}^{\text {hyd }}=G_{\text {ele }}^{\text {hyd }}+G_{\text {non-ele }}^{\text {hyd }}$. Changes in these two terms based on temperature are shown in SI. It is known that the $G_{\text {non-ele }}^{\text {hyd }}$ term is composed by dispersion 


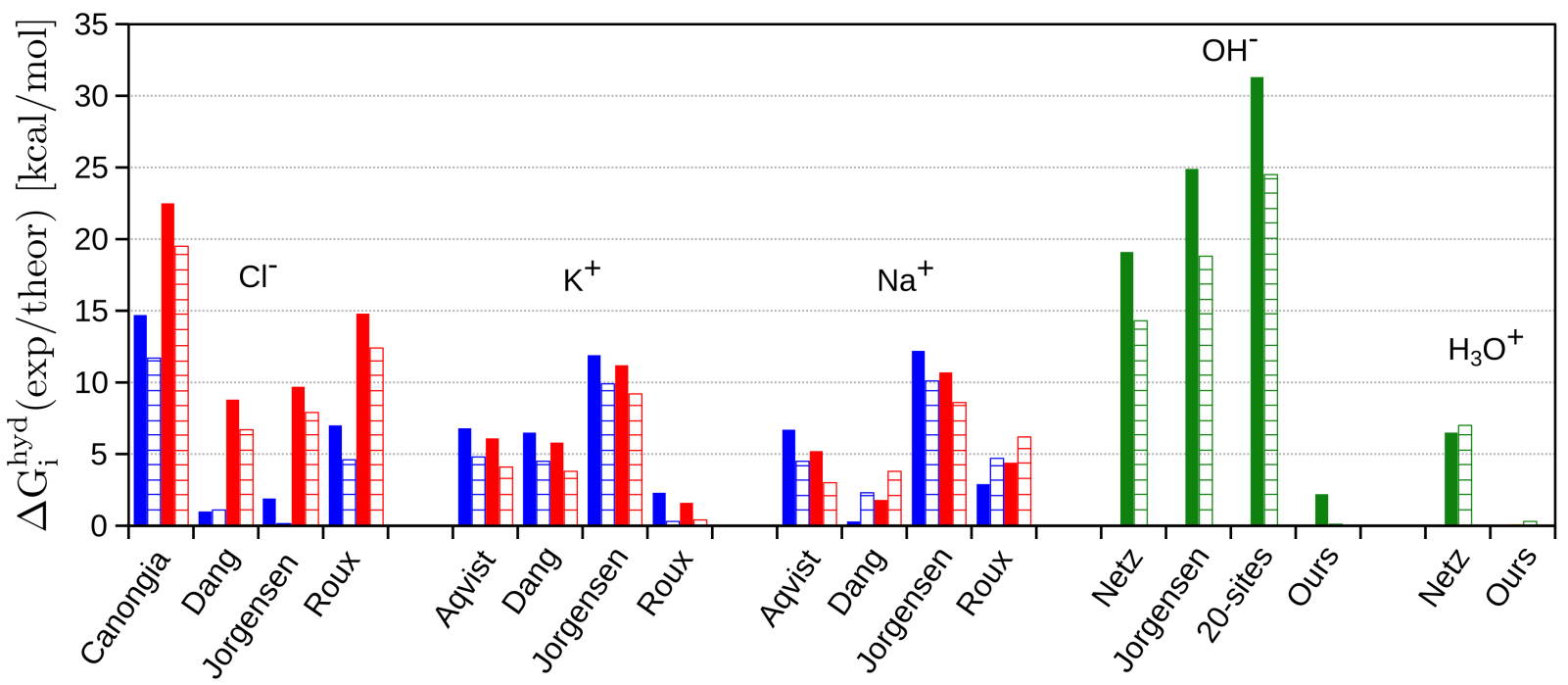

Figure 1: Experimental/theoretical differences of the hydration free energy, $\Delta G_{i}^{\text {hyd }}$ (exp/theor) at $T=298 \mathrm{~K}$ for different ionic force field parameters with two water models: SPC/E (solid bar) and TIP3P (open bar). The colors represent different experimental data presented by: Schmid et al. ${ }^{38}$ (blue), Yizhak ${ }^{39}$ (red) and Pliego and Riveros $^{40}$ (green).

(always negative) and cavitation (always positive) contributions for the free energy. For all ions, the cavitation contribution is dominant, once it results in a positive $G_{n o n-e l e}^{\text {hyd }}$. This term, in particular, has decreased sensitivity to temperature change and lower magnitude from 0.1 to $5.9 \mathrm{kcal} / \mathrm{mol}$ for monoatomic ions, 0.8 to $4.6 \mathrm{kcal} / \mathrm{mol}$ for aqueous ions, and 1.8 to $8.6 \mathrm{kcal} / \mathrm{mol}$ for alkyl ions by considering all force field parameters. For the latter two, $G_{\text {non-ele }}^{\text {hyd }}$ is proportional to the size of the chain, which is larger for $T B A^{+}$than $T M A^{+}$. On the other hand, the term $G_{\text {ele }}^{\text {hyd }}$ is negative for all ions. It has greater sensitivity to temperature change and it is larger in magnitude than $G_{\text {non-ele }}^{\text {hyd }}$, i.e. from - 64.8 to $-107.2 \mathrm{kcal} / \mathrm{mol}$ for monoatomic ions, -103.5 to $-139.9 \mathrm{kcal} / \mathrm{mol}$ for aqueous ions and -22.1 to $-39.8 \mathrm{kcal} / \mathrm{mol}$ for alkyl ions, again considering all force field parameters. Changes in $G_{i}^{\text {hyd }}(T)$ due to different temperatures arise predominantly from electrostatic interactions. $G_{\text {ele }}^{\text {hyd }}$ increases as follows: $\mathrm{TBA}^{+}<\mathrm{TMA}^{+}<\mathrm{K}^{+}<\mathrm{Na}^{+}<\mathrm{Cl}^{-}<\mathrm{OH}^{-}<\mathrm{H}_{3} \mathrm{O}^{+}$for force field parameters that reached best experimental/theoretical agreement at room temperature.

The solvation distribution of water molecules around ions has been analyzed by the radial 

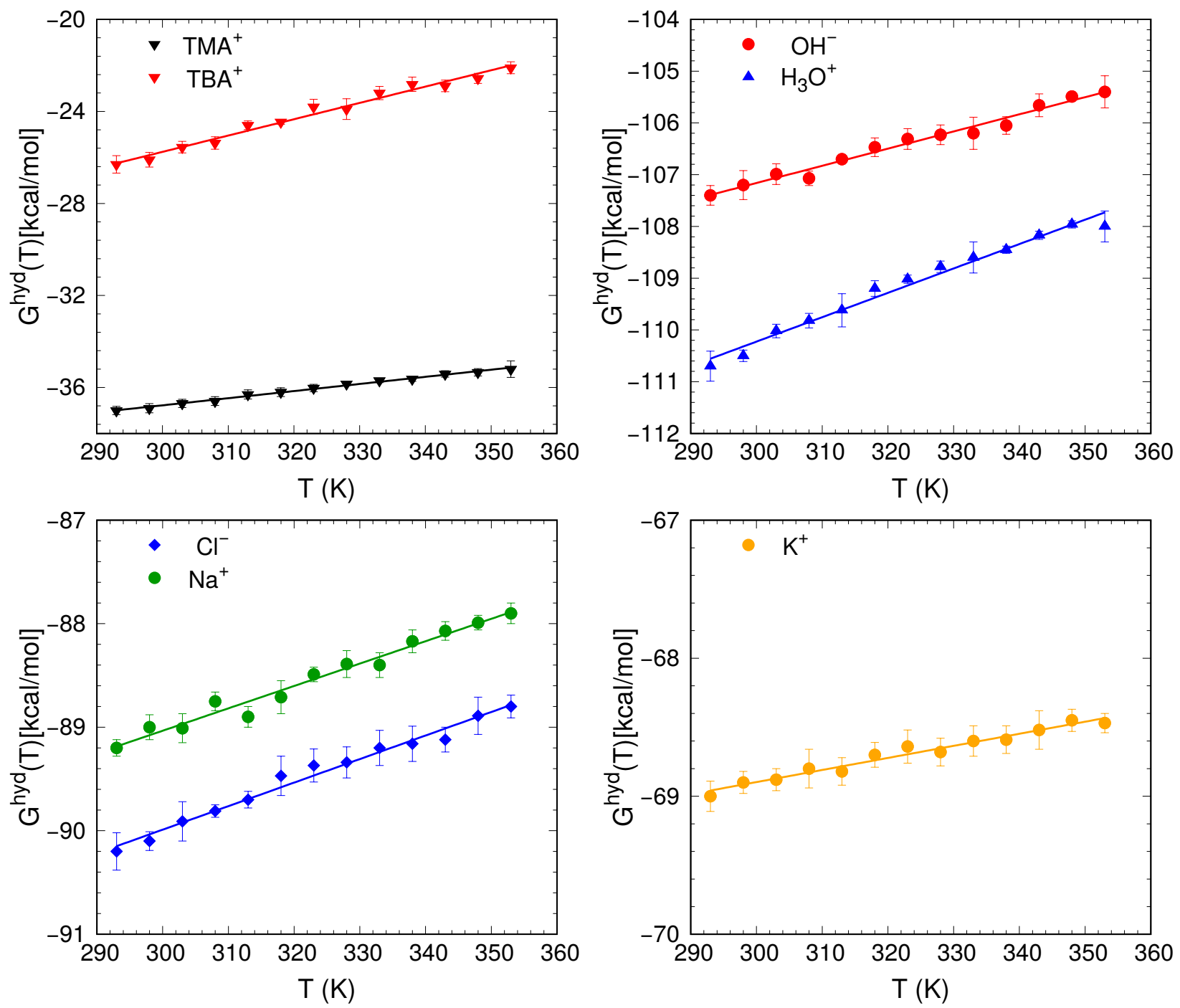

Figure 2: Hydration free energy $G_{i}^{\text {hyd }}(T)$ calculated for ions at temperatures ranging between 293 to $353 \mathrm{~K}$ using the BAR method in MD simulations using force field parameters with best experimental/theoretical agreement (Dang for $\mathrm{Cl}^{-}$and $\mathrm{Na}^{+}$, Roux for $\mathrm{K}^{+}$and Ours for $\mathrm{OH}^{-}, \mathrm{H}_{3} \mathrm{O}^{+}, \mathrm{TMA}^{+}$and $\mathrm{TB} \mathrm{A}^{+}$) and the SPC/E water model. The best linear fit is presented for each ion and its slope describes the ionic hydration entropy (in cal $/ \mathrm{mol} \cdot \mathrm{K}$ ), $-S_{i}^{\text {hyd }}=d G_{i}^{\text {hyd }}(T) / d T=8.4 \pm 0.5$ for $K^{+}$(bottom right with yellow circle), $20.8 \pm 1.1$ for $\mathrm{Na}^{+}$and $24.2 \pm 0.9$ for $\mathrm{Cl}^{-}$(bottom left with green circle and blue diamond, respectively), $32.2 \pm 1.5$ for $\mathrm{OH}^{-}$and $35.4 \pm 2.0$ for $\mathrm{H}_{3} \mathrm{O}^{+}$(top right with red circle and blue triangle, respectively), $30.8 \pm 0.8$ for $T M A^{+}$and $72.4 \pm 3.0$ for $T B A^{+}$(top left with black and red down triangles, respectively).

distribution function, $G(r)$, at different temperatures (Figures are shown in SI). As expected, specific interactions between water molecules and monoatomic or aqueous ions can be easily identified at distances of around 0.2-0.3 nm comprising the first maximum of $G(r)$ between 
ions and the atoms of Oxygen and Hydrogen in water, i.e. ion-OW and ion-HW. In the case of negative ions, the $G_{\text {ion-HW }}(r)$ peak is closer (around $0.1 \mathrm{~nm}$ ) than the $G_{\text {ion-OW }}(r)$ peak, while the $G_{\text {ion-OW }}(r)$ peak is closer in the case of positive ions while describing Hydrogen bond formation. However, in the case of alkyl ions, $G_{\text {ion-OW }}(r)$ and $G_{\text {ion-HW }}(r)$, peaks appear at the same position (around 0.4-0.5 nm) showing a tangential orientation of the $O H$ bond of water molecules. Thus no specific interactions with water molecules are found around $T M A$ and $T B A$ ions indicating clathrate formation. $G(r)$ presents very small differences with respect to temperature changes. Tables showing distances of the beginning, maximum and first minimum of $G(r)$ and the coordination numbers of ions with temperature ranging from $293 \mathrm{~K}$ to $353 \mathrm{~K}$ are shown in SI. Differences that are smaller than $0.02 \mathrm{~nm}$ were observed for the first peak and the largest variation of the coordination number was found in a reduction of less than 1.6 water molecules $\left(<10 \%\right.$ of the first solvation shell) for $T M A^{+}$ and $T B A^{+}$when temperature increased from $293 \mathrm{~K}$ to $353 \mathrm{~K}$. Therefore, it is concluded that the temperature effect in the range of $293 \mathrm{~K}$ and $353 \mathrm{~K}$ is negligible in the solvation structure of water molecules around ions.

An increase in $G_{i}^{h y d}$ for rising temperatures means that the cold side is preferred by ions diffusing in a temperature gradient. The difference $\Delta G_{i}^{h y d}(T)=G_{i}^{h y d}(T+\delta T)-G_{i}^{h y d}(T)$ in an initially homogeneous ionic solution with instantaneously applied temperature gradient is due to the maximum probability density shift along time, as the ion moves towards the position of lower free energy. The success of using physical parameters obtained from the thermodynamic equilibrium for describing non-equilibrium effects has been discussed in literature. ${ }^{29,37}$ It is a probabilistic point of view that explains the reasons for increasing ions concentration in one side of a temperature gradient, which Fick law of diffusion assumes a similar due to the induced concentration gradient. It is worth mentioning that the directional diffusion phenomena expressed by Fick equation for diffusion with a drift term ${ }^{53}$ modulates inhomogeneity in concentration induced by the temperature gradient. Therefore, the amount of possible ions that migrates towards the cold side is defined by the difference 
$\Delta G_{i}^{h y d}(T)$, but the time to stabilize concentration distribution depends on the self-diffusion constant, which is to be discussed as follows.

\subsection{Ion and salt diffusion}

The rate of ion displacement is related to the shift in the maximum probability density towards a position with lower free energy in a temperature gradient and is quantified by the selfdiffusion coefficient, $D_{i} \cdot{ }^{37}$ By calculating the slope of the ionic Mean Square Displacement (MSD) through MD simulations at different temperatures (the same temperatures shown in Table 1), values for $D_{i}(T)$ were obtained, which are shown in Table 2 for ionic force field parameters with the best experimental/theoretical agreement of $G_{i}^{\text {hyd }}(298)$. For comparison purposes, some experimental values ${ }^{8,41}$ available in literature are also presented. The values of $D_{i}(T)$ obtained from MD simulations are in good agreement with experimental data with less than $0.09 \times 10^{-5} \mathrm{~cm}^{2} / \mathrm{s}$ ) of difference, except for hydroxide and hydronium ions whose calculated values are highly underestimated at room temperature $\left(2.17\right.$ and $\left.2.45 \times 10^{-5} \mathrm{~cm}^{2} / \mathrm{s}\right)$ for $\mathrm{OH}^{-}$and $\mathrm{H}_{3} \mathrm{O}^{+}$, respectively, in comparison with 5.32 and $9.31 \times 10^{-5} \mathrm{~cm}^{2} / \mathrm{s}$ ) obtained experimentally). However, it is well known in literature that the diffusion of hydroxide and hydronium in aqueous solution occurs predominantly by a Grotthus-type mechanism involving proton exchange between water molecules. ${ }^{54}$ This mechanism is not considered in classical MD simulations, because water molecules are not able to protonate/deprotonate in classical MD simulations. It requires more sophisticated methodologies, such as first-principles MD simulations, among other techniques. ${ }^{55,56}$ Due to such a limitation of MD simulations, we decided to use the experimental values found in literature ${ }^{41}$ for $\mathrm{OH}^{-}$and $\mathrm{H}_{3} \mathrm{O}^{+}$at different temperatures (showed in Table 2) for further calculations in this work.

By raising temperatures from 293 to $353 \mathrm{~K}$, it was found a diffusion coefficient increase between 2.1 times for the $\mathrm{H}_{3} \mathrm{O}^{+}$ion and up to 3.5 times for $\mathrm{Na}^{+}$ion. At room temperature, the calculated self-diffusion coefficient $D_{i}(298)$ shows that ions follow the same experimental tendency: ${ }^{8} T B A^{+}<T M A^{+}<N a^{+}<K^{+}<\mathrm{Cl}^{-}<O H^{-}<H_{3} O^{+}$, in which the $T B A^{+}$ 
Table 2: Self-diffusion coefficients of ions, $D_{i}(T)$ (in $10^{-5} \mathrm{~cm}^{2} / \mathrm{s}$ ), in aqueous solution calculated using MD simulations at different temperatures using force field parameters with best experimental/theoretical agreement (Dang for $\mathrm{Cl}^{-}$and $\mathrm{Na}^{+}$, Roux for $\mathrm{K}^{+}$and Ours for $\mathrm{OH}^{-}, \mathrm{H}_{3} \mathrm{O}^{+}, \mathrm{TMA}^{+}$and $\mathrm{TB} A^{+}$) and the SPC/E water model. Experimental values are in brackets.

\begin{tabular}{cccccc}
\hline Ion & $D_{i}(293)$ & $D_{i}(298)$ & $D_{i}(313)$ & $D_{i}(333)$ & $D_{i}(353)$ \\
\hline $\mathrm{Cl}^{-}$ & 1.45 & $2.12[2.03]^{a}$ & 2.55 & 3.06 & 3.48 \\
$\mathrm{~K}^{+}$ & 1.27 & $1.89[1.96]^{a}$ & 2.17 & 3.11 & 4.19 \\
$\mathrm{Na}^{+}$ & 0.95 & $1.45[1.33]^{a}$ & 1.77 & 2.38 & 3.33 \\
$\mathrm{OH}^{-}$ & {$[4.76]^{b}$} & $2.17[5.32]^{a}$ & {$[7.03]^{b}$} & {$[9.55]^{b}$} & {$[12.41]^{b}$} \\
$\mathrm{H}_{3} \mathrm{O}^{+}$ & {$[8.50]^{b}$} & $2.45[9.31]^{a}$ & {$[11.72]^{b}$} & {$[14.99]^{b}$} & {$[18.22]^{b}$} \\
$\mathrm{TMA}^{+}$ & 0.91 & $1.15[1.20]^{a}$ & 1.34 & 2.14 & 2.54 \\
$\mathrm{TBA}^{+}$ & 0.38 & $0.49[0.51]^{a}$ & 0.66 & 0.79 & 1.21 \\
\hline
\end{tabular}

${ }^{a}$ Evaluated values taken from experimental measurements, reference $\left[{ }^{8}\right] .{ }^{b}$ Evaluated values taken from experimental measurements of ionic conductivity, reference $\left[{ }^{41}\right]$. To convert ionic conductivity, $C_{i}(T)$, into ion self-diffusion $D_{i}(T)$, the Nerst-Einstein equation was used: $D_{i}(T)=R T C_{i}(T) / F^{2}$, where $R$ is the ideal gas constant, $T$ is temperature and $F$ is the Faraday constant. The standard deviation of calculated $D_{i}(T)$ is lower than $0.10 \times 10^{-5} \mathrm{~cm}^{2} / \mathrm{s}$.

is the slowest ion and $\mathrm{H}_{3} \mathrm{O}^{+}$is the fastest ion in aqueous solution. An increase in $D_{i}$ values from temperature differences is expected due to increased thermal energy and decreased water viscosity. Temperature gradients in aqueous electrolytes mean that the self-diffusion coefficient is position-dependent, that has been assigned as a source of thermophoretic migration in the temperature gradient. ${ }^{57}$ This effect is, in principle, not related to the single-ion Soret effect.

Once the values of ion self-diffusion coefficients $D_{i}$ (showed in Table 2) are obtained, the effective mass diffusion $D$ of salts has been calculated for different temperatures using the following equation ${ }^{7}$

$$
D=\frac{2 D_{+} D_{-}}{D_{+}+D_{-}}
$$

The calculated values of $D(T)$ and experimental data ${ }^{16,42}$ are shown in Figure 3 . All values are presented in SI. There is a good agreement between calculated and experimental values for all salts. The calculated values are in the range of 0.7 and $5.9 \times 10^{-5} \mathrm{~cm}^{2} / \mathrm{s}$ 
and experimental values range from 0.6 to $5.3 \times 10^{-5} \mathrm{~cm}^{2} / \mathrm{s}$. The dependence of effective mass diffusion $D(T)$ on temperature for both sets of data presents a linear growth as temperature increases for all salts. The smallest slope is $0.02 \times 10^{-5} \mathrm{~cm}^{2} / \mathrm{sK}$ obtained for
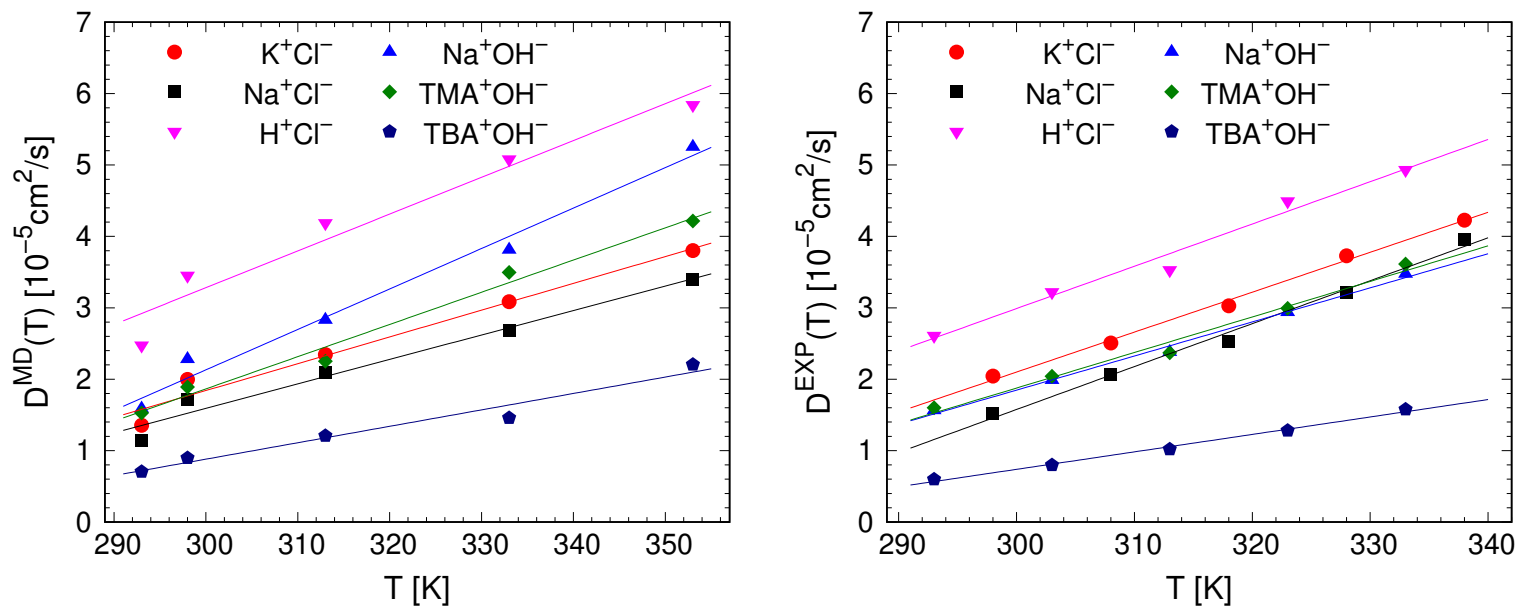

Figure 3: Effective mass diffusion $D(T)$ (in $10^{-5} \mathrm{~cm}^{2} / \mathrm{s}$ ) for different temperatures: (left) calculated $D^{M D}(T)$ using Equation 3 with ion self-diffusion coefficients $D_{i}$ showed in Table 2 and (right) experimental $D^{E X P}(T)$ obtained from optical experiments. ${ }^{16,42}$ The symbols and colors represent different salts: $\mathrm{K}^{+} \mathrm{Cl}^{-}$(red circle), $\mathrm{Na}^{+} \mathrm{Cl}^{-}$(black square), $\mathrm{H}^{+} \mathrm{Cl}^{-}$(pink down triangle), $\mathrm{Na}^{+} \mathrm{OH}^{-}$(blue triangle), $\mathrm{TMA}^{+} \mathrm{OH}^{-}$(green diamond) and $\mathrm{TMA}^{+} \mathrm{OH}^{-}$ (dark blue pentagon).

$\mathrm{TBA}^{+} \mathrm{OH}^{-}$in both sets and the slopes of other salts $\left(\mathrm{K}^{+} \mathrm{Cl}^{-}, \mathrm{Na}^{+} \mathrm{Cl}^{-}, \mathrm{H}^{+} \mathrm{Cl}^{-}, \mathrm{Na}^{+} \mathrm{OH}^{-}\right.$ and $\mathrm{TMA}^{+} \mathrm{OH}^{-}$) are between 0.05 and $0.06 \times 10^{-5} \mathrm{~cm}^{2} / \mathrm{sK}$ for experimental sets and between 0.03 and $0.06 \times 10^{-5} \mathrm{~cm}^{2} / \mathrm{sK}$ for calculated sets. The most pronounced difference was found in the $D$ slope of $\mathrm{Na}^{+} \mathrm{Cl}^{-}, 0.03 \times 10^{-5} \mathrm{~cm}^{2} / \mathrm{sK}$ for calculated values and $0.06 \times 10^{-5} \mathrm{~cm}^{2} / \mathrm{sK}$ for experimental data. All the linear regressions are presented in SI. $\mathrm{TBA}^{+} \mathrm{OH}^{-}$has the lowest effective diffusion, $\mathrm{H}^{+} \mathrm{Cl}^{-}$has the highest effective diffusion and the other salts have an intermediate diffusion. These behaviors reflect the values of ion self-diffusion coefficients $D_{i}$ (see Table 2), where the $T B A^{+}$ion has the smallest $D_{i}(298)=0.51 \times 10^{-5} \mathrm{~cm}^{2} / \mathrm{s}$ and the $\mathrm{H}_{3} \mathrm{O}^{+}$ion has the largest $D_{i}(298)=9.31 \times 10^{-5} \mathrm{~cm}^{2} / \mathrm{s}$. 


\subsection{Ion thermodiffusion}

The single-ion heat of transport $Q_{i}^{*}$ and the single-ion Soret coefficient $\alpha_{i}$ were calculated from the dependence of the ion hydration free energy $G_{i}^{h y d}(T)$ on temperature. Then, by using $\alpha_{i}$ and the ion self-diffusion coefficient $D_{i}(T)$, it was calculated the ion mass current density $j_{T i}$ that describes the thermodiffusion of ions in aqueous solution. In Figure 2, it is shown a linear dependence of $G_{i}^{h y d}(T)$ on temperature and a positive slope for all ions in the range of $T=293$ to $353 \mathrm{~K}$. Therefore, the angular coefficient of the linear best fit of calculated $G_{i}^{\text {hyd }}(T)$ is constant and positive in this temperature range, and was used to calculate the modules of $S_{i}^{h y d}(T)$. Furthermore, it was obtained the value of modules of the single-ion heat of transport $Q_{i}^{*}$ using two approaches: (i) the direct calculation from the MD simulation, $Q_{i}^{*}(\mathrm{MD})=-T S_{i}^{\text {hyd }}$; and (ii) the scaling factor of Takeyama and Nakashima, ${ }^{31}$ $Q_{i}^{*}(\mathrm{MD} / \mathrm{TN})=-f_{i}^{T N} T S_{i}^{h y d}$ where the scaling factor $f_{i}^{T N}$ is 0.348 for $\mathrm{Cl}^{-}, 0.383$ for $\mathrm{OH}^{-}$, 0.439 for $\mathrm{Na}^{+}, 0.450$ for $\mathrm{H}_{3} \mathrm{O}^{+}, 0.457$ for $\mathrm{K}^{+}$, and 0.555 for $\mathrm{TMA}^{+}$and $\mathrm{TBA}^{+}$.

In Figure 4, we compared these two sets of calculated values, $Q_{i}^{*}(\mathrm{MD})$ and $Q_{i}^{*}(\mathrm{MD} / \mathrm{TN})$, for different ionic force field parameters and two water models (SPC/E and TIP3P) with the experimental values obtained by Agar et al., ${ }^{8} Q_{i}^{*}$ (Agar). It was observed the best agreement between experimental/theoretical values, $\Delta Q_{i}^{*}(\exp /$ theor), for the $\mathrm{MD} / \mathrm{TN}$ procedure (green bars) showing that the scaling factor $f_{i}^{T N}$ improves the calculated values of $Q_{i}^{*}(\mathrm{MD} / \mathrm{TN})$ in comparison with $Q_{i}^{*}$ (Agar). As a general trend, both water models (solid bars for $\mathrm{SCP} / \mathrm{E}$ and open bars for TIP3P) present similar values, but with SPC/E model the $\Delta Q_{i}^{*}(\exp /$ theor $)$ values are slightly smaller than with TIP3P. Therefore, for further discussions, we will present only the results obtained with $\mathrm{SPC} / \mathrm{E}$ water model (the results for TIP3P are shown in SI), but it is important to note all conclusion are valid for both water models. Another interesting observation concerning $Q_{i}^{*}$ is that there is a good performance of the same force

field parameters that reached best experimental/theoretical agreement for $G_{i}^{\text {hyd }}(T)$ : Dang for $\mathrm{Cl}^{-}$and $\mathrm{Na}^{+}$, Roux for $\mathrm{K}^{+}$and Ours for $\mathrm{OH}^{-}, \mathrm{H}_{3} \mathrm{O}^{+}, \mathrm{TMA}^{+}$and $\mathrm{TB} \mathrm{A}^{+}$. They present $\Delta Q_{i}^{*}(\exp /$ theor $)<2.5 \mathrm{kcal} / \mathrm{mol}$, except for $T B A^{+}$with $\Delta Q_{i}^{*}(\exp /$ theor $)>3.5 \mathrm{kcal} / \mathrm{mol}$. 
However, Jorgensen force field parameters for monoatomic ions present a slightly better performance in comparison due to the better temperature dependence of $G_{i}^{h y d}(T)$ for $C l^{-}$, $\mathrm{Na}^{+}$and $\mathrm{K}^{+}$.

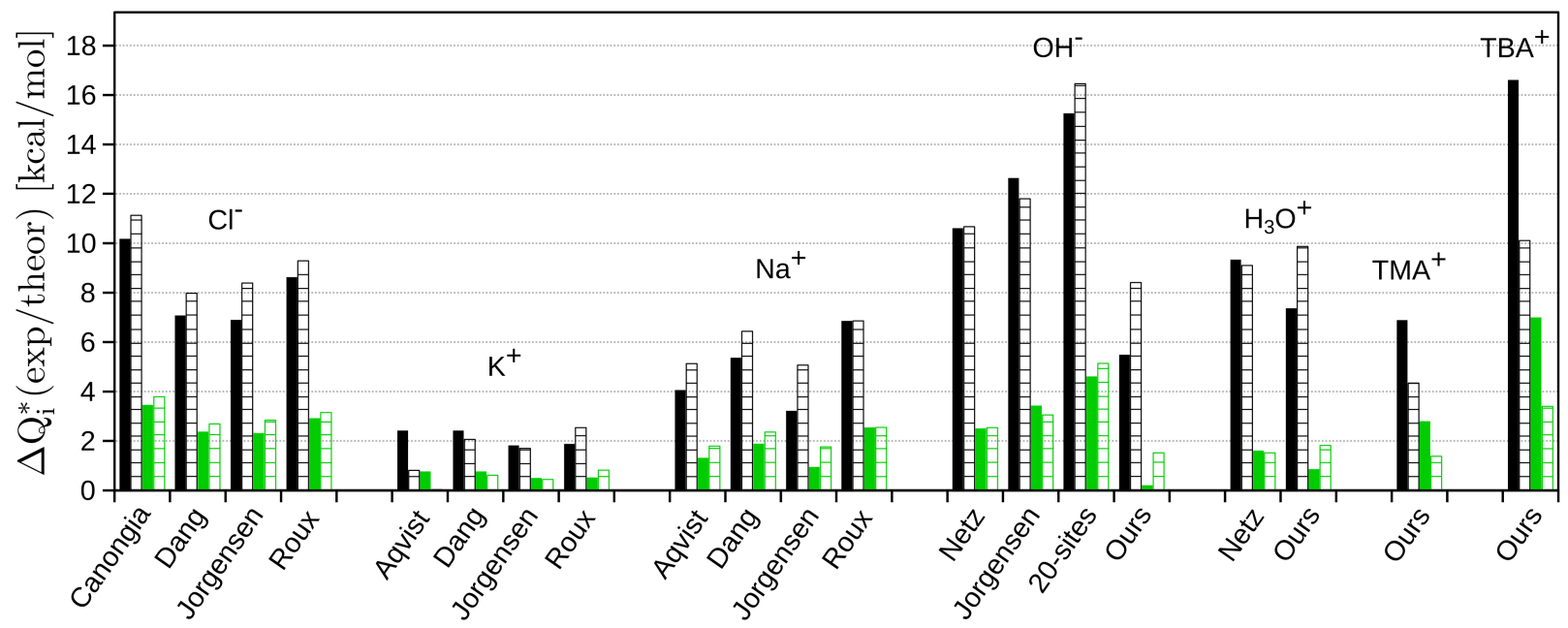

Figure 4: Experimental/theoretical differences of the single-ion heat of transport, $\Delta Q_{i}^{*}(\exp /$ theor $)$ at $T=298 \mathrm{~K}$ for different ionic force field parameters with two water models: SPC/E (solid bar) and TIP3P (open bar). The colors represent two theoretical procedures MD (black) and MD/TN (green) in comparison with the experimental data obtained by Agar et al. ${ }^{8}$

Table 3 shows the experimental data, ${ }^{8} Q_{i}^{*}$ (Agar), and the two sets of theoretical values, $Q_{i}^{*}(\mathrm{MD})$ and $Q_{i}^{*}(\mathrm{MD} / \mathrm{TN})$ obtained by $\mathrm{MD}$ simulations with different ionic force field parameters and the $\mathrm{SPC} / \mathrm{E}$ water model at $T=298 \mathrm{~K}$. The values obtained with the TIP3P water model are shown in SI.

Using Equation 2, we obtained the corresponding sets of single-ion Soret coefficients $\alpha_{i}$ values: $\alpha_{i}(\mathrm{MD}), \alpha_{i}(\mathrm{MD} / \mathrm{TN})$ and $\alpha_{i}$ (Agar). By comparing $Q_{i}^{*}$ and $\alpha_{i}$ shown in Table 3 , it is possible to observe that the three sets of values present a similar trend: the values for polyatomic ions are higher than those for monoatomic ions. The values of $Q_{i}^{*}($ Agar $)$ and $\alpha_{i}$ (Agar) increase as follows $\mathrm{Cl}^{-}<\mathrm{K}^{+}<\mathrm{Na}^{+}<T M A^{+}<\mathrm{H}_{3} \mathrm{O}^{+}<\mathrm{OH}^{-}<T B A^{+}$.

As for $\mathrm{OH}^{-}$and $\mathrm{H}_{3} \mathrm{O}^{+}$, the best calculated values for $\alpha_{i}(\mathrm{MD} / \mathrm{TN})$ are 3.65 and 3.41 which are in very good agreement with $\alpha_{i}$ (Agar) values, 3.48 and 2.70, respectively, using our force field parameters. As regards $K^{+}, N a^{+}, T M A^{+}$and $T B A^{+}$, the best calculated values for 
$\alpha_{i}(\mathrm{MD} / \mathrm{TN})$ are $0.94,1.50,4.30$ and 10.11 , that are around two times larger than $\alpha_{i}($ Agar $)$ values, $0.52,0.70,2.02$ and 4.21, respectively, using Jorgensen force field parameters for monoatomic ions and our parameters for polyatomic ions. $\mathrm{Cl}^{-}$is the only ion that the best calculated value, $\alpha_{C l^{-}}(\mathrm{MD} / \mathrm{TN})=2.07$, is far from $\alpha_{C l^{-}}$(Agar) $=0.11$ by a factor of twenty times. Therefore, we believe that new force field parameters for $\mathrm{Cl}^{-}$should be developed in the future to better describe the heat of transport and Soret coefficient. The current force field parameters are able to reproduce the hydration free energy of $\mathrm{Cl}^{-}$(the highest experimental value) and self-diffusion coefficient, as shown in Tables 1 and 2, respectively. But they fail to describe its derivative with respect to temperature, $\Delta G_{i}^{\text {hyd }}(T) / \Delta T$.

Our results show that the presence of temperature gradient in salted water induces an increasing ion concentration towards the cold side of the solution for all studied cations and anions due to the hydration free energy $G_{i}^{h y d}(T)$ temperature dependence. Therefore, the single-ion Soret coefficient $\alpha_{i}$ together with the rate of ion displacement described by the self-diffusion coefficient $D_{i}$ generate different intensities of ion flux given by Equation 1. Assuming that an experimental setup can be prepared using the same values of the numerical volumetric concentration of ions $n_{i}$ and temperature gradient $\nabla T$ for all ions, we analyzed the reduced mass current density $j_{T i} / c=\alpha_{i} D_{i}$, where $c=-n_{i} \nabla T / T$. The calculated values of $j_{T i} / c$ at room temperature are shown in Table 3 . The values of ion flux intensity $j_{T i} / c($ Agar) increase as follows $\mathrm{Cl}^{-}<\mathrm{K}^{+}<\mathrm{Na}^{+}<\mathrm{TB} A^{+}<\mathrm{TM} A^{+}<\mathrm{OH}^{-}<\mathrm{H}_{3} \mathrm{O}^{+}$. Then, by comparing this sequence with $\alpha_{i}$ (Agar) sequence $\left(\mathrm{Cl}^{-}<\mathrm{K}^{+}<\mathrm{Na}^{+}<\mathrm{TM} A^{+}<\mathrm{H}_{3} \mathrm{O}^{+}<\right.$ $\left.O H^{-}<T B A^{+}\right)$and $D_{i}$ sequence $\left(T B A^{+}<T M A^{+}<N a^{+}<K^{+}<C l^{-}<O H^{-}<H_{3} O^{+}\right)$, it is possible to conclude that the flux of monoatomic ions is dominated by single-ion Soret coefficients, while the flux of polyatomic ions is dominated by self-diffusion coefficients. This information is important, since it can be used to plan ions combination that may generate salts with better thermodiffusion effect. 
Table 3: Single-ion heat of transport $Q_{i}^{*}$ (in $\mathrm{kcal} / \mathrm{mol}$ ) calculated at $T=298 \mathrm{~K}$ using MD and MD/TN approaches. In brackets are the values obtained experimentally by Agar et al., ${ }^{8}$ $Q_{i}^{*}$ (Agar). Single-ion Soret coefficient $\alpha_{i}$ (Equation 2) and single-ion reduced mass current density $j_{T i} / c$ (Equation 1 ) were calculated with three sets of $Q_{i}^{*}$.

\begin{tabular}{|c|c|c|c|c|c|c|c|c|c|c|}
\hline \multirow[b]{2}{*}{ Ion } & \multirow[b]{2}{*}{ Force Field } & \multicolumn{3}{|c|}{$Q_{i}^{*}$} & \multicolumn{3}{|c|}{$\alpha_{i}$} & \multicolumn{3}{|c|}{$j_{T i} / c=\alpha_{i} D_{i}$} \\
\hline & & MD & $\mathrm{MD} / \mathrm{TN}$ & Agar & $\mathrm{MD}$ & $\mathrm{MD} / \mathrm{TN}$ & Agar & $\mathrm{MD}$ & $\mathrm{MD} / \mathrm{TN}$ & Agar \\
\hline \multirow{4}{*}{$C l^{-}$} & Canongia & 10.31 & 3.59 & \multirow{4}{*}[0.13]{} & 8.71 & 3.03 & \multirow{4}{*}{0.11} & 18.46 & 6.42 & \multirow{4}{*}{0.23} \\
\hline & Dang & 7.21 & 2.51 & & 6.09 & 2.12 & & 12.91 & 4.49 & \\
\hline & Jorgensen & 7.03 & 2.45 & & 5.94 & 2.07 & & 12.59 & 4.38 & \\
\hline & Roux & 8.76 & 3.05 & & 7.40 & 2.57 & & 15.68 & 5.46 & \\
\hline \multirow{4}{*}{$K^{+}$} & Aqvist & 3.04 & 1.39 & \multirow{4}{*}[0.62]{} & 2.57 & 1.17 & \multirow{4}{*}{0.52} & 4.85 & 2.22 & \multirow{4}{*}{0.98} \\
\hline & Dang & 3.04 & 1.39 & & 2.57 & 1.17 & & 4.85 & 2.22 & \\
\hline & Jorgensen & 2.44 & 1.12 & & 2.06 & 0.94 & & 3.90 & 1.78 & \\
\hline & Roux & 2.50 & 1.14 & & 2.11 & 0.97 & & 3.99 & 1.83 & \\
\hline \multirow{4}{*}{$N a^{+}$} & Aqvist & 4.89 & 2.15 & \multirow{4}{*}[0.83]{} & 4.13 & 1.81 & \multirow{4}{*}{0.70} & 5.98 & 2.63 & \multirow{4}{*}{1.02} \\
\hline & Dang & 6.20 & 2.72 & & 5.23 & 2.30 & & 7.59 & 3.33 & \\
\hline & Jorgensen & 4.05 & 1.78 & & 3.42 & 1.50 & & 4.96 & 2.18 & \\
\hline & Roux & 7.69 & 3.38 & & 6.49 & 2.85 & & 9.41 & 4.13 & \\
\hline \multirow{4}{*}{$O H^{-}$} & Netz & 14.72 & 6.62 & \multirow{4}{*}[4.11]{} & 12.43 & 5.59 & \multirow{4}{*}{3.48} & 66.13 & 29.76 & \multirow{4}{*}{18.51} \\
\hline & Jorgensen & 16.75 & 7.54 & & 14.14 & 6.36 & & 75.23 & 33.85 & \\
\hline & 20 -sites & 19.37 & 8.72 & & 16.35 & 7.36 & & 87.01 & 39.15 & \\
\hline & Ours & 9.60 & 4.32 & & 8.10 & 3.65 & & 43.10 & 19.40 & \\
\hline \multirow{2}{*}{$\mathrm{H}_{3} \mathrm{O}^{+}$} & Netz & 12.52 & 4.79 & \multirow{2}{*}[3.18]{} & 10.57 & 4.05 & \multirow{2}{*}{2.70} & 98.38 & 37.68 & \multirow{2}{*}{25.14} \\
\hline & Ours & 10.55 & 4.04 & & 8.91 & 3.41 & & 82.92 & 31.76 & \\
\hline$T M A^{+}$ & Ours & 9.18 & 5.09 & [2.39] & 7.75 & 4.30 & 2.02 & 8.91 & 4.95 & 2.32 \\
\hline$T B A^{+}$ & Ours & 21.58 & 11.97 & {$[4.97]$} & 18.22 & 10.11 & 4.21 & 8.93 & 4.95 & 2.06 \\
\hline
\end{tabular}

\subsection{Thermodiffusion of salts}

In a thermodiffusion analysis, the stationary state of the concentration gradient is considered, i.e. the total ionic flux is null. Then, $j_{T}+j_{n}=0$, where $j_{T}=-n D_{T} \nabla T, j_{n}=-D \nabla n, D_{T}$ is the salt thermal diffusion coefficient and $D$ is the effective mass diffusion coefficient. In the case where positive and negative ions are not distinguished, the salt Soret coefficient is simply defined as $S_{T}=D_{T} / D$. The values of $S_{T}$ and $D$ are experimentally accessible from amplitudes and transients, respectively, of optical experiments ${ }^{16,42}$ in which the probe volume 
of samples are infinitely larger in comparison with ionic sizes. However, by distinguishing anions $\left(\alpha_{-}\right.$and $\left.D_{-}\right)$and cations $\left(\alpha_{+}\right.$and $\left.D_{+}\right)$, the stationary state has additional relations, ${ }^{7}$ such as:

$$
S_{T}=\frac{\alpha_{+}+\alpha_{-}}{T}
$$

and Equation 3. As both descriptions should be equivalent, it is possible to find the following relation

$$
D_{T}=\frac{D\left(\alpha_{+}+\alpha_{-}\right)}{T}
$$

Therefore, using Equations 4 and 5, we calculate the salt Soret coefficient $S_{T}$ and the salt thermal diffusion coefficient $D_{T}$, respectively. In addition, we performed a discussion about their temperature dependence and a comparison with experimental values obtained with optical measurements. ${ }^{16,42}$

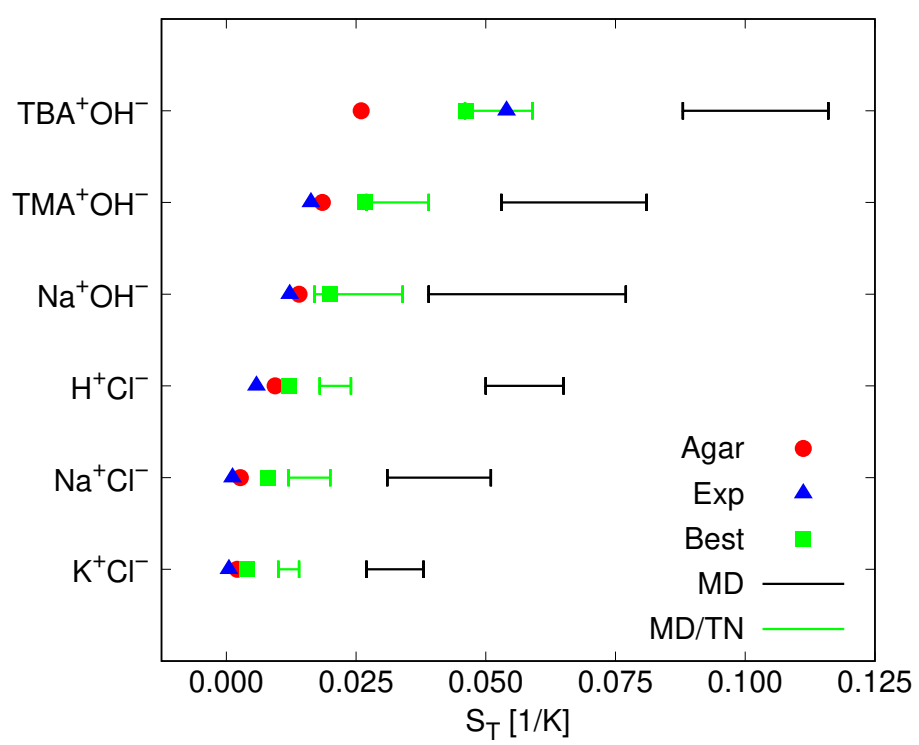

Figure 5: Salt Soret coefficient $S_{T}$ (in 1/K) calculated using Equation 4 with single-ion Soret coefficient $\alpha_{i}$ showed in Table 3 and experimental data obtained by optical measurements. ${ }^{16,42}$ The colors represent: $S_{T}$ (Agar) (red), $S_{T}(\mathrm{MD})$ (black), $S_{T}(\mathrm{MD} / \mathrm{TN})$ (green) and $S_{T}(\operatorname{Exp})$ (blue). The horizontal bars represent the range obtained using MD simulations with different ionic force field parameters and the SPC/E water model. The green square with legend "Best" represents $S_{T}(\mathrm{MD} / \mathrm{TN})$ values calculated using the force field parameters with best experimental/theoretical agreement (Dang for $\mathrm{Cl}^{-}$and $\mathrm{Na}^{+}$, Roux for $\mathrm{K}^{+}$and Ours for $\mathrm{OH}^{-}, \mathrm{H}_{3} \mathrm{O}^{+}, \mathrm{TMA}^{+}$and $\mathrm{TB} A^{+}$) and the SPC/E water model, but with modified $\mathrm{Cl}^{-}$ Soret coefficient, $\alpha_{C l^{-}}^{B e s t}=\alpha_{C l^{-}} / 10$. 
Figure 5 shows the salt Soret coefficient $S_{T}$ calculated using single-ion Soret coefficients $\alpha_{i}$ (Table 3 ) at $T=298 \mathrm{~K}$ in comparison with experimental data. ${ }^{16,42}$ The values are presented in Table 4 for force field parameters with the best experimental/theoretical agreement. As expected, the values of $S_{T}$ obtained from $\alpha_{i}^{*}($ Agar $)$ are closer to experimental results, since they were calculated from $Q_{i}^{*}($ Agar $)$ obtained through calorimetric experiments assuming a infinite dilution, ${ }^{8}$ except for the $T B A^{+} O H^{-}$salt where the experimental value $S_{T}(\operatorname{Exp})=$ $5.40 \times 10^{-2} 1 / \mathrm{K}$ is almost twice the $S_{T}($ Agar $)=2.60 \times 10^{-2} 1 / \mathrm{K}$. The calculated values for $S_{T}$ obtained through MD simulations using different force field parameters for ions are indicated as intervals (horizontal bars) in Figure 5 and all individual values are shown in SI. The two sets of calculated $S_{T}$, with $\mathrm{MD}$ and $\mathrm{MD} / \mathrm{TN}$, are in the same order of magnitude as the experimental data, but the approach $\mathrm{MD} / \mathrm{TN}$ provides values closer to the experimental data. Both sets present a similar trend if compared to the experimental data: the values for salts composed of polyatomic ions $\left(\mathrm{OH}^{-}, \mathrm{H}_{3} \mathrm{O}^{+}, \mathrm{TMA}^{+}\right.$and $\left.\mathrm{TB} \mathrm{A}^{+}\right)$are larger than those for salts composed of monoatomic ions $\left(\mathrm{Cl}^{-}, \mathrm{K}^{+}\right.$and $\left.\mathrm{Na}^{+}\right)$. It can be seen that the scaling factor $f_{i}^{T N}$ that relates the single-ion heat of transport to the ion hydration entropy proposed by Takeyama and Nakashima, ${ }^{31}$ improves the calculated values of salt Soret coefficient, $S_{T}(\mathrm{MD} / \mathrm{TN})$, in comparison with experimental data, $S_{T}(\operatorname{Exp})$. Analyzing the correlation between calculated and experimental values of $S_{T}$ presented in Table 4, a linear regression of $S_{T}(\operatorname{Exp})=1.55 S_{T}(\mathrm{MD} / \mathrm{TN})-2.04$ with $R^{2}=96 \%$ was obtained, hence showing a very good correlation between both sets of data. An interesting additional observation is that the overestimated value of calculated $\alpha_{C l^{-}}(\mathrm{MD} / \mathrm{TN})$ imposes larger calculated values of $S_{T}$ for chloride salts $\left(\mathrm{K}^{+} \mathrm{Cl}^{-}, \mathrm{Na}^{+} \mathrm{Cl}^{-}\right.$and $\left.\mathrm{H}^{+} \mathrm{Cl}^{-}\right)$, once $S_{T}$ is proportional to $\alpha_{+}+\alpha_{-}$. Thus, a better parametrization of the $\mathrm{Cl}^{-}$force field can improve the agreement of calculated $S_{T}(\mathrm{MD} / \mathrm{TN})$ values with the experimental data even further. This hypothesis was assessed by dividing the $\alpha_{C l^{-}}(\mathrm{MD} / \mathrm{TN})$ by a factor of 10 , i.e. $\alpha_{C l^{-}}^{B e s t}=\alpha_{C l^{-}} / 10$, and recalculating the $S_{T}$ for chloride salts showed in Figure 5 and in parenthesis on Table 4.

A broadly discussed topic in thermodiffusion is the temperature dependence of the salt 
Table 4: Salt Soret coefficient $S_{T}\left(\right.$ in $\left.\times 10^{-2} 1 / \mathrm{K}\right)$ showed in Figure 5 and fast and slow Seebeck coefficients, $S_{\text {fast }}$ and $S_{\text {slow }}$ (in $\mathrm{mV} / \mathrm{K}$ ) showed in Figure 7 . MD/TN values were obtained by using ionic force field parameters with best experimental/theoretical agreement (Dang for $\mathrm{Cl}^{-}$and $\mathrm{Na}^{+}$, Roux for $\mathrm{K}^{+}$and Ours for $\mathrm{OH}^{-}, \mathrm{H}_{3} \mathrm{O}^{+}, \mathrm{TMA}^{+}$and $\mathrm{TBA} \mathrm{A}^{+}$) and the SPC/E water model. In parenthesis are values calculated using $\alpha_{\mathrm{Cl}^{-}}^{\text {Best }}$.

\begin{tabular}{cccccccccc}
\hline & \multicolumn{3}{c}{$S_{T}$} & \multicolumn{3}{c}{$S_{\text {fast }}$} & \multicolumn{3}{c}{$S_{\text {slow }}$} \\
Salt & MD/TN & Agar & Exp $^{42}$ & MD/TN & Agar & Exp $^{43}$ & MD/TN & Agar & Exp $^{43}$ \\
\hline$K^{+} C l^{-}$ & 1.04 & 0.21 & 0.05 & 0.10 & 0.04 & 0.30 & 0.10 & 0.01 & 0.17 \\
& $(0.40)$ & & & $(0.06)$ & & & $(0.07)$ & & \\
$N a^{+} C l^{-}$ & 1.48 & 0.27 & 0.12 & 0.06 & 0.03 & 0.50 & 0.02 & 0.03 & 1.60 \\
& $(0.80)$ & & & $(0.13)$ & & & $(0.18)$ & & \\
$H^{+} \mathrm{Cl}^{-}$ & 1.86 & 0.94 & 0.58 & 0.42 & 0.46 & 0.90 & 0.11 & 1.10 & 2.00 \\
& $(1.20)$ & & & $(0.48)$ & & & $(0.28)$ & & \\
$\mathrm{Na}^{+} \mathrm{OH}^{-}$ & 1.99 & 1.40 & 1.22 & 0.42 & 0.43 & 0.30 & 0.12 & 1.30 & 1.10 \\
$\mathrm{TMA}^{+} \mathrm{OH}^{-}$ & 2.67 & 1.85 & 1.63 & 0.38 & 0.38 & 0.50 & 0.06 & 0.83 & 1.20 \\
$\mathrm{TBA}^{+} \mathrm{OH}^{-}$ & 4.62 & 2.60 & 5.40 & 0.42 & 0.48 & 0.20 & 0.56 & 2.10 & 3.60 \\
\hline
\end{tabular}

Soret coefficient $S_{T}$ and the salt thermal diffusion coefficient $D_{T} \cdot{ }^{18,19,23-25,33,35,42}$ Using the relation between $D_{T}$ and $\alpha_{i}$ (Equation 5), the temperature dependence of the salt thermal diffusion coefficient $D_{T}(T)$ was analyzed. Note that the calculated single-ion Soret coefficients $\alpha_{i}$ for all salts were obtained as constant and positive at temperatures ranging from 293 to $353 \mathrm{~K}$. Then, we used the calculated effective mass diffusion coefficient $D$ (showed in Figure 3) obtained for different temperatures to calculate $D_{T}(T)$. These results of $D_{T}(T)$ calculated through $\alpha_{i}(\mathrm{MD} / \mathrm{TN})$ are shown in Figure 6 together with the experimental data ${ }^{16,42}$ for comparison purposes. All values are presented in SI. There was a good agreement between the calculated and experimental values for all salts. The best agreement is for $\mathrm{TB} \mathrm{A}^{+} \mathrm{OH}^{-}$where the calculated values are in the range of 0.032 to $0.067 \times 10^{-5} \mathrm{~cm}^{2} / \mathrm{sK}$ at temperatures from 293 to $333 \mathrm{~K}$, and experimental values are in the range of 0.026 to $0.074 \times 10^{-5} \mathrm{~cm}^{2} / \mathrm{sK}$ for the same temperature range. The linear temperature dependence seen for calculated $D_{T}^{M D / T N}(T)$ resembles the linear dependence found in the experimental data. The slope of $D_{T}^{M D / T N}(T)$ ranges from 0.38 to $1.21 \times 10^{-8} \mathrm{~cm}^{2} / \mathrm{sK}$ and $D_{T}^{E X P}(T)$ ranges from 0.27 to $1.14 \times 10^{-8} \mathrm{~cm}^{2} / \mathrm{sK}$, in which $\mathrm{K}^{+} \mathrm{Cl}^{-}$and $\mathrm{Na}^{+} \mathrm{Cl}^{-}$have the lowest values $\left(\sim 0.45 \times 10^{-8} \mathrm{~cm}^{2} / \mathrm{sK}\right.$ for $D_{T}^{M D / T N}(T)$ 
and $\sim 0.30 \times 10^{-8} \mathrm{~cm}^{2} / \mathrm{sK}$ for $\left.D_{T}^{E X P}(T)\right)$ and $T M A^{+} O H^{-}$and $T B A^{+} O H^{-}$achieved the highest values $\left(\sim 1.10 \times 10^{-8} \mathrm{~cm}^{2} / \mathrm{sK}\right.$ for $D_{T}^{M D / T N}(T)$ and $\sim 1.05 \times 10^{-8} \mathrm{~cm}^{2} / \mathrm{sK}$ for $\left.D_{T}^{E X P}(T)\right)$. This analysis reveals that the temperature dependence of thermodiffusion coefficient $D_{T}(T)$ is just a consequence of the temperature dependence of the ratio $D(T) / T$.
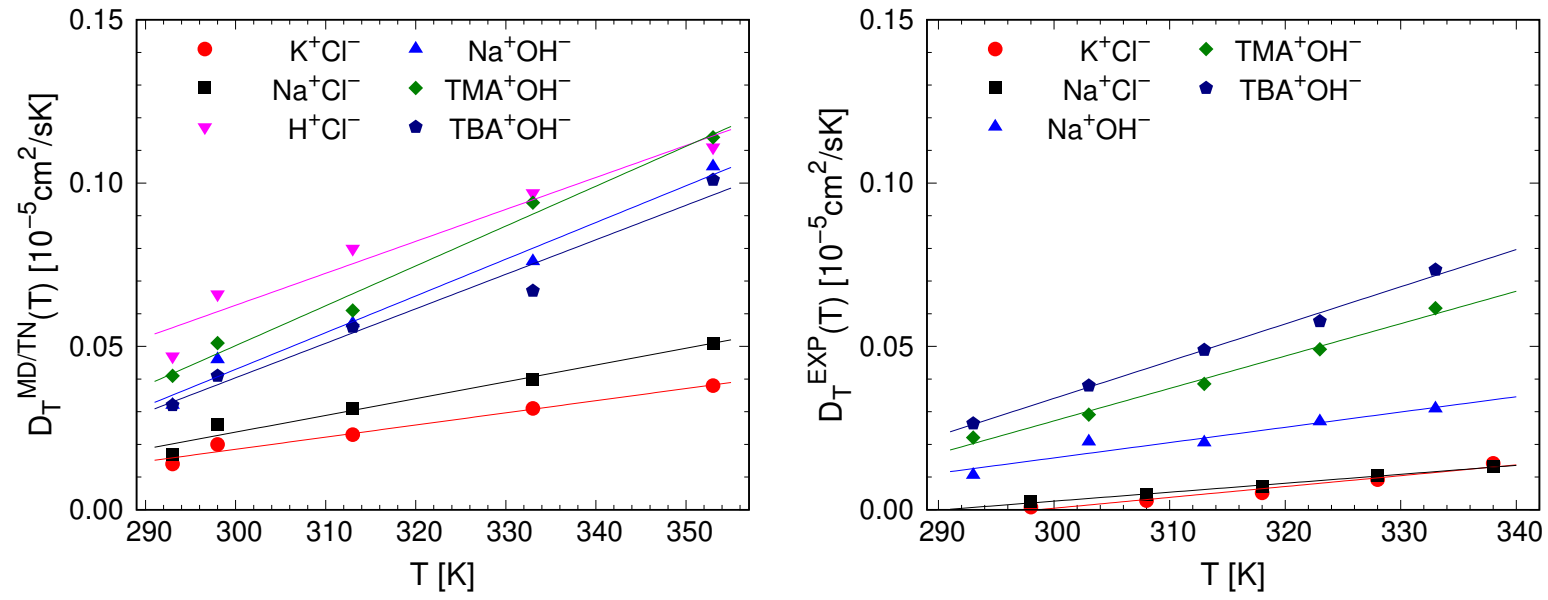

Figure 6: Thermodiffusion coefficient $D_{T}(T)$ (in $10^{-5} \mathrm{~cm}^{2} / \mathrm{sK}$ ) for different temperatures: (left) calculated $D_{T}^{M D / T N}(T)$ using Equation 5 with single-ion Soret coefficients $\alpha_{i}(\mathrm{MD} / \mathrm{TN})$ showed in Table 3 and effective mass diffusion $D(T)$ showed in Figure 3, and (right) experimental $D_{T}^{E X P}(T)$ obtained from optical experiments. ${ }^{16,42}$ The symbols and colors represent different salts: $\mathrm{K}^{+} \mathrm{Cl}^{-}$(red circle), $\mathrm{Na}^{+} \mathrm{Cl}^{-}$(black square), $\mathrm{H}^{+} \mathrm{Cl}^{-}$(pink down triangle), $\mathrm{Na}^{+} \mathrm{OH}^{-}$(blue triangle), $\mathrm{TMA}^{+} \mathrm{OH}^{-}$(green diamond) and $\mathrm{TMA}^{+} \mathrm{OH}^{-}$(dark blue pentagon).

Different values of the single-ion Soret coefficients $\alpha_{i}$ for anions and cations mean that one kind of ion has a trend to flow more than the other one in temperature gradients. To avoid charge separation, a thermoelectric field arises in solution. This is usually described in a phenomenological way as the generalized equations for ion current density: ${ }^{7}$

$$
j_{i}=-D_{i} n_{i}\left(\frac{\partial \ln n_{i}}{\partial x}-\frac{z_{i} e}{k_{B} T} E_{x}+2 \alpha_{i} \frac{\partial \ln T}{\partial x}\right),
$$

where $z_{i}$ is the valence number, $x$ is a space coordinate and $E_{x}$ is the hypothetical ad hoc thermoelectric field. Recently, it was shown that $E_{x}$ arises from two dynamical effects that are distant in at least 8 orders of magnitude in their transient times: ${ }^{22} E_{1}$ proportional to 
the difference of products $\alpha_{+} D_{+}-\alpha_{-} D_{-}$, which affect the fast response of the system that is much longer than the Debye transient and much shorter than the diffusive transient; and $E_{2}$ proportional to the difference $\alpha_{+}-\alpha_{-}$, which affects the slow response of the system that is much longer than the diffusive transient. ${ }^{22}$ The equations for amplitude modules of Seebeck coefficients related to both time transients are given by: ${ }^{22,58}$

$$
S_{f a s t}=\frac{2 k_{B}}{e}\left|\frac{\alpha_{+} D_{+}-\alpha_{-} D_{-}}{D_{+}+D_{-}}\right|
$$

and

$$
S_{\text {slow }}=\frac{k_{B}}{e}\left|\alpha_{+}-\alpha_{-}\right|
$$

In Figure 7, we show the calculated modules of fast and slow Seebeck coefficients, $S_{\text {fast }}$ and $S_{\text {slow }}$, using Equations 7 and 8 with self-diffusion coefficient $D_{i}$ and single-ion Soret coefficients $\alpha_{i}$ showed in Tables 2 and 5, respectively. All values of different force field parameters for ions and water molecules are presented in SI. Table 4 presents the values obtained using MD/TN approximation for simulations with force field parameters with best experimental/theoretical agreement and the SPC/E water model. All values for the other force field are presented in SI. The calculated values for $S_{\text {fast }}$ obtained from the single-ion Soret coefficient $\alpha_{i}(\mathrm{MD} / \mathrm{TN})$ and $\alpha_{i}($ Agar $)$ are close to the experimental data. ${ }^{43} S_{\text {fast }}(\mathrm{MD} / \mathrm{TN})$ values are in the range of 0.06 to $0.42 \times 10^{-2} 1 / \mathrm{K}$ and experimental values are in the range of 0.2 to $0.9 \times 10^{-2} 1 / \mathrm{K}$.

A comparison between calculated and experimental values are shown in Figure 7(left). We found a large correlation between $S_{\text {fast }}(\mathrm{MD} / \mathrm{TN})$ and $S_{\text {fast }}\left(\right.$ Agar) with $R^{2}=96 \%$, which shows that the self-diffusion of anions and cations, $D_{-}$and $D_{+}$, dominate the fast response of the thermoelectric field, since $S_{\text {fast }}(\mathrm{MD} / \mathrm{TN})$ and $S_{\text {fast }}($ Agar) were calculated using the same set of $D_{i}$ values showed in Table 2 . No correlation between $S_{\text {fast }}(\mathrm{MD} / \mathrm{TN})$, or $S_{\text {fast }}($ Agar $)$, and the experimental data was observed, $R^{2}<10 \%$. It means that although the values predicted by the DM/TN approach and the single-ion heat of transport $Q_{i}^{*}$ experimentally 

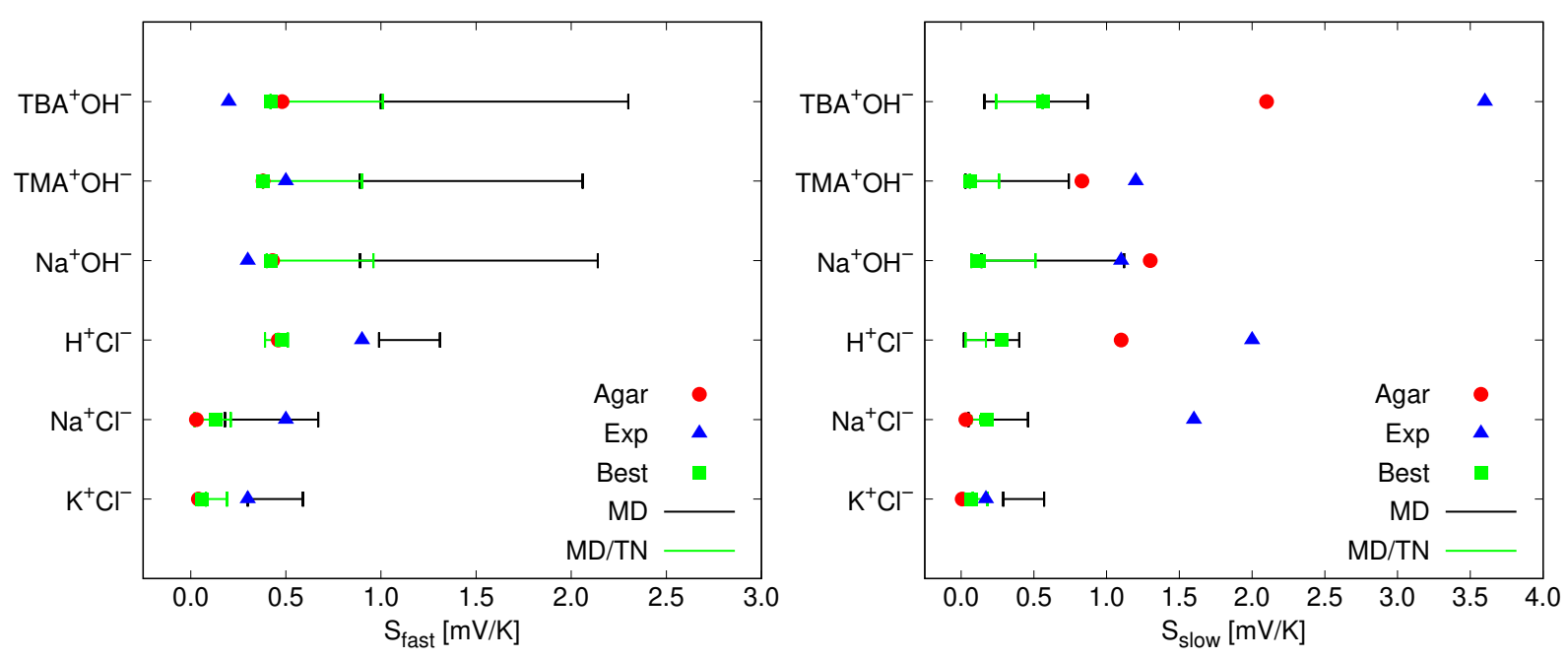

Figure 7: Modules of fast and slow Seebeck coefficients, $S_{\text {fast }}$ (left) and $S_{\text {slow }}$ (right), (in $\mathrm{mV} / \mathrm{K}$ ) calculated using Equations 7 and 8, respectively, with self-diffusion coefficients $D_{i}$ showed in Table 2 and single-ion Soret coefficient $\alpha_{i}$ showed in Table 3, and the experimental data obtained with optical measurements. ${ }^{43}$ The colors and symbols descriptions are the same as shown in Figure 5.

determined by Agar et al. ${ }^{8}$ are in the same order of magnitude as the experimental data, they can not predict the fast thermoelectric response. Nevertheless, in general, there is still a lack of experimental results for the fast thermoelectric field of salts in aqueous solution so as to establish definite conclusions.

On the other hand, a theoretical prediction using calculated values of $S_{\text {slow }}$ is more important, since it describes the system after reaching the thermoelectric equilibrium long after the ionic diffusion has taken place. A comparison between calculated and experimental values are shown in Figure 7(right). Attention ought to be given to the order of magnitude for calculated Seebeck coefficients, as it is the same as the one obtained from experiments involving aqueous electrolytes. ${ }^{1,2,59} S_{\text {slow }}(\mathrm{MD} / \mathrm{TN})$ values are in the range of 0.02 to $0.56 \mathrm{mV} / \mathrm{K}$ and experimental values are in the range of 0.17 to $3.60 \mathrm{mV} / \mathrm{K}$. Therefore, $S_{\text {slow }}(\mathrm{MD} / \mathrm{TN})$ presents lower values than the $S_{\text {slow }}(\operatorname{Exp})$. However, we found a linear correlation between $S_{\text {slow }}(\mathrm{MD} / \mathrm{TN})$ and $S_{\text {slow }}(\operatorname{Exp})$ with $R^{2}=66 \%$ which has been improved when $\alpha_{C l^{-}}^{B e s t}$ was used, $R^{2}=90 \%$. Figure 8 shows this last correlation with $R^{2}=90 \%$ between $S_{\text {slow }}(\mathrm{MD} / \mathrm{TN})$ using $\alpha_{C l^{-}}^{B e s t}$ and $S_{\text {slow }}(\operatorname{Exp})$ and additionally the correlation between $S_{\text {slow }}($ Agar $)$ and $S_{\text {slow }}(\operatorname{Exp})$ 
with $R^{2}=61 \%$. For $S_{\text {slow }}$ (Agar), it can be seen that the values for $\mathrm{Na}^{+} \mathrm{Cl}^{-}$(open red triangle) and $\mathrm{Na}^{+} \mathrm{OH}^{-}$(solid red triangle) present large deviation with respect to the linear fit (dashed red line) causing a weak correlation between them, but its slope is near 1.0 showing values of $S_{\text {slow }}$ (Agar) close to the experimental data $S_{\text {slow }}(\operatorname{Exp})$ as shown in Table 4 . On the other hand, for $S_{\text {slow }}(\mathrm{MD} / \mathrm{TN})$ all calculated values (black symbols) are close to the linear fit (dashed black line) given a strong correlation between experimental/theoretical values. Therefore, theoretical MD/TN procedure can predict the experimental values of Seebeck coefficient through the following relation: $S_{\text {slow }}(\operatorname{Exp})=5.79 S_{\text {slow }}(\mathrm{MD} / \mathrm{TN})+0.39$.

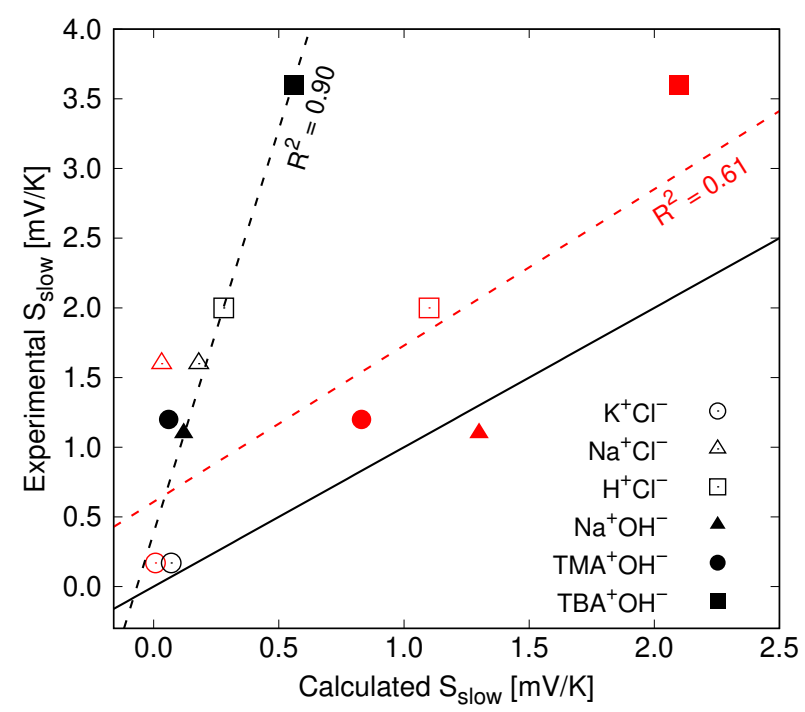

Figure 8: Relation between calculated and experimental modules of the slow Seebeck coefficient (in $\mathrm{mV} / \mathrm{K}): S_{\text {slow }}(\mathrm{MD} / \mathrm{TN})$ (black symbols) and $S_{\text {slow }}($ Agar) (red symbols) showed in Table 4. The symbols represent different salts: $\mathrm{K}^{+} \mathrm{Cl}^{-}$(open circle), $\mathrm{Na}^{+} \mathrm{Cl}^{-}$(open triangle), $\mathrm{H}^{+} \mathrm{Cl}^{-}$(open square), $\mathrm{Na}^{+} \mathrm{OH}^{-}$(solid triangle), $\mathrm{TMA}^{+} \mathrm{OH}^{-}$(solid circle) and $T \mathrm{MA}^{+} \mathrm{OH}^{-}$(solid square). The dashed lines represent the linear relation of $S_{\text {slow }}(\operatorname{Exp})=$ $5.79 S_{\text {slow }}(\mathrm{MD} / \mathrm{TN})+0.39$ with $R^{2}=90 \%$ (black) and $S_{\text {slow }}(\operatorname{Exp})=1.12 S_{\text {slow }}($ Agar $)+$ 0.61 with $R^{2}=61 \%$ (red). The solid black line represents a complete agreement between calculated and experimental values.

The theoretical approach proposed herein using MD simulations and the scaling factor $f_{i}^{T N}$ between the hydration entropy for ions $S_{i}^{h y d}$ and the single-ion heat of transport $Q_{i}^{*}$ is capable of predicting the Seebeck coefficient and describing the thermodiffusion of salts in aqueous solution. We suggest that this approach is suitable to be used in the description of 
experimental results, such as those obtained by Zhao et al. ${ }^{9}$ and Li et al., ${ }^{2}$ who had performed experimental measurements of aqueous electrolytes with optimized interfaces or porousconfining media to generate strong, unpredictable and unprecedented Seebeck coefficients. As recently remarked by Zhao et al., ${ }^{9}$ a good strategy to obtain a high thermoelectric response is to use electrolytes with high self-diffusion difference between constituents. ${ }^{1,3,60}$ A further step is to confirm that the high values of $\Delta D=D_{+}-D_{-}$are only partly responsible for the high Seebeck coefficients values. The results lead us to state that a fundamental condition is a strong variation of ionic hydration free energy $G_{i}^{h y d}(T)$ for dispersed solute (like salts and polyelectrolytes) in water solution. In other words, high values of $\Delta \alpha=\alpha_{+}-\alpha_{-}$or/and $\Delta\left(\alpha_{i} D_{i}\right)=\alpha_{+} D_{+}-\alpha_{-} D_{-}$render high values of Seebeck coefficient $S$, mainly due to the slow thermodiffusion effect. Thus, our method can be used to theoretically verify the feasibility of new materials to be inserted into ionic solutions so as to present high Soret or Seebeck coefficients, i.e. before carrying out time-consuming tests in laboratory.

\section{Conclusion}

An analysis of the ionic hydration free energy temperature dependence $G_{i}^{h y d}(T)$ obtained from MD simulations in water solution resulted in a temperature-constant ionic hydration entropy $S_{i}^{h y d}$, ionic heat of transport $Q_{i}$ and single-ion Soret coefficients $\alpha_{i}$, which show a trend of good agreement with experimental results. All studied anions and cations prefer the cold side of the temperature gradient, i.e. they have positive single-ion Soret coefficient. Polyatomic ions, such as $\mathrm{OH}^{-}, \mathrm{H}_{3} \mathrm{O}^{+}, \mathrm{TBA}^{+}$and $\mathrm{TM} A^{+}$, present greater temperature dependence, thus resulting in a higher single-ion Soret coefficient $\alpha_{i}$, while monoatomic ions, such as $\mathrm{Na}^{+}, \mathrm{K}^{+}$and $\mathrm{Cl}^{-}$, present lower temperature dependence due to achieving decreased $\alpha_{i}$. Moreover, our analysis has revealed that most contributions for the temperature dependence of $G_{i}^{h y d}(T)$ are from the electrostatic ion-water interaction. However, for alkyl ions $\left(T B A^{+}\right.$and $\left.T M A^{+}\right)$, the non-electrostatic ion-water interaction, i.e. the Van der Waals or 
Lennard-Jones interaction, plays an important role at around $40 \%$ of $G_{i}^{\text {hyd }}(T)$ variation. By comparing our results to the experimental data, it was found that a better agreement was obtained by using the scaling factor $f_{i}^{T N}$ between the single-ion heat of transport $Q_{i}^{*}$ and the ionic hydration entropy $S_{i}^{h y d}$ proposed by Takeyama and Nakashima ${ }^{31}\left(f_{i}^{T N}=0.348\right.$ for $\mathrm{Cl}^{-}, 0.383$ for $\mathrm{OH}^{-}, 0.439$ for $\mathrm{Na}^{+}, 0.450$ for $\mathrm{H}_{3} \mathrm{O}^{+}, 0.457$ for $\mathrm{K}^{+}$, and 0.555 for $\mathrm{TMA}^{+}$ and $\left.T B A^{+}\right)$. The calculated values of ion flux intensity $j_{T i} / c=\alpha_{i} D_{i}$ increase as follows $\mathrm{Cl}^{-}<\mathrm{K}^{+}<\mathrm{Na}^{+}<\mathrm{TB} A^{+}<\mathrm{TMA} A^{+}<\mathrm{OH}^{-}<\mathrm{H}_{3} \mathrm{O}^{+}$, where the flux of monoatomic ions is dominated by single-ion Soret coefficients $\alpha_{i}$, while the flux of polyatomic ions is dominated by self-diffusion coefficients $D_{i}$.

From the calculated single-ion Soret coefficient $\alpha_{i}$, the salt Soret $S_{T}$ and fast and slow Seebeck, $S_{\text {fast }}$ and $S_{\text {slow }}$, coefficients were calculated, which also show good agreement with experimental results. We discuss the specific topic of thermodiffusion, i.e. the ionic temperature dependence on the thermal diffusion coefficient $D_{T}(T)$, which shows that $D_{T}(T)$ depends on the ratio $D(T) / T$, where $D(T)$ is the salt effective mass diffusion coefficient.

The simulation reported in this work was performed at infinite ionic dilutions, i.e. adding more water molecules to the simulated box did not change the final result for $G_{i}^{h y d}(T)$. We proposed a consistent approach that allowed comparison with experiments, taking advantage of the existing connection between the equilibrium hydration free energy $G_{i}^{\text {hyd }}$ and the nonequilibrium effect. A comparison between experimental data and results obtained with non-equilibrium MD simulations with a temperature gradient along the simulation box, is not straightforward. Given the fact that MD simulations use thermostatic methods, most of which were formulated to describe the stage of temperature equilibrium and they have no realistic physical behavior in the non-equilibrium stage. Then, these thermostatic methods provide an unrealistic ionic flux through the temperature gradient.

In our point of view, the limitations of the theoretical approach proposed in this work to calculate thermodiffusion coefficients are the inherent limitations of classical molecular simulations that are related to a good parameterization of ions in order to adequately describe 
the temperature dependence of the hydration, or solvation, free energy and the impossibility to describe the self-diffusion of hydroxide and hydronium in aqueous solution occurs predominantly by a Grotthus-type mechanism involving proton exchange between water molecules. But, this last limitation was avoided using the experimental values of the hydroxide and hydronium self-diffusion coefficients at different temperatures. Our theoretical approach may

be used to estimate $G_{i}^{h y d}(T)$ of thermoelectric materials and predict the slow Seebeck responses, in addition to an intuitive use of materials with a large difference in self-diffusion and single-ion Soret coefficients obtained from pure electrolyte aqueous solutions. The possibility to perform MD simulations with large ionic concentration and connect the results with transport coefficients is desired for applications in thermoelectric devices and biological systems, usually those dispersed in ionic aqueous solution.

\section{Methodology}

We have performed two types of molecular dynamics simulations using the GROMACS package: ${ }^{61}$ (i) the conventional dynamics (cMD) with Newton equation of motion coupled with a stochastic thermostat to generate the ion-water trajectories, analyze the solvation shells around ions and calculate the ion self diffusion coefficient; and (ii) the stochastic dynamics (sMD) with Langevin equation of motion with small friction constant to calculate ion hydration free energy. These two methods are equivalent, ${ }^{61,62}$ but sMD becomes more advantageous in the calculation of the free energy variations due to the enhanced conformational search ability. ${ }^{62}$ In both types, the simulated system was composed of an ion $\left(\mathrm{Na}^{+}, \mathrm{K}^{+}\right.$, $\mathrm{Cl}^{-}, \mathrm{OH}^{-}, \mathrm{H}_{3} \mathrm{O}^{+}, \mathrm{TMA}^{+}$or $\mathrm{TB} \mathrm{A}^{+}$), a counter-ion $\left(\mathrm{Na}^{+}\right.$or $\mathrm{Cl}^{-}$) surrounded by 1000 (or 2000) water molecules in a cubic box (for the case of organic ions $T M A^{+}$and $T B A^{+}$). The simulations were performed in a NPT ensemble at $P=1$ atm at different temperatures in the range of 293 and $353 \mathrm{~K}$. The NPT ensemble was obtained using Velocity Rescaling thermostat $^{63}$ for temperature control in cMD simulations with a coupling constant of $0.1 \mathrm{ps,}$ 
and a Berendsen barostat ${ }^{64}$ for pressure control with a coupling constant of 2 ps. In sMD, the temperature control is obtained directly from the Langevin equation of motion with the friction constant of $1.0 \mathrm{ps}^{-1}$. All interactions were computed inside a cut-off-radius of $14 \AA$. A long-range correction for electrostatic interactions was treated with the smooth particlemesh Ewald method ${ }^{65}$ with cubic interpolation and Fourier spacing of $14 \AA$. The equations of motion were integrated using the leapfrog algorithm ${ }^{66}$ in $\mathrm{cMD}$ simulations and its stochastic version $^{67}$ in sMD simulations, and implemented as $s d$ integrator in GROMACS package. ${ }^{68}$ In both types of simulations, it was used a time step of $1 \mathrm{fs}$ with constraints in all H-bonds using the LINCS algorithm. ${ }^{69}$ The center of mass motion was linearly removed for the whole system at each 200 fs. For cMD simulations, 5 ns were performed in the thermalization stage and $10 \mathrm{~ns}$ in the equilibrium stage.

In order to obtain the ionic hydration free energy $G_{i}^{\text {hyd }}$, non-bonding interaction energies between the ion and the solvent were multiplied by a $\lambda$ scaling factor $(0<\lambda<1)$, where $\lambda=0$ means totally turned off interactions and $\lambda=1$ means totally turned on interactions. Therefore, the solvation process was performed in two stages: first, we used a set of $11 \lambda_{L J}$ values with $\lambda_{q}=0$ to create the Lennard-Jones (LJ) particle-water interaction and then with $\lambda_{L J}=1$ we used a set of $11 \lambda_{q}$ values to create the ion-water Coulomb interaction in the preexistent LJ particle. The set of 11 values used for $\lambda_{L J}$ and $\lambda_{q}$ are $\{0.0,0.1,0.2,0.3,0.4,0.5$, $0.6,0.7,0.8,0.9$ and 1.0$\}$. To avoid problems in the initial configuration, for each simulation with $\lambda_{L J}$ and $\lambda_{q}$, we started with a thermalized configuration obtained from a previous cMD simulation with $\lambda_{L J}=1$ and $\lambda_{q}=1$, and then 1 ns were performed in the thermalization stage and $2 \mathrm{~ns}$ in the equilibrium stage for sMD simulations. Therefore, in order to calculate the differences of hydration free energy $G_{\lambda_{i}}^{\text {hyd }}$, the potential energy differences between each sMD simulation with neighboring $\lambda$ were obtained through the Bennet Acceptance Ratio (BAR) method ${ }^{70}$ using the gmx bar command available in the GROMACS package. The sum of free energy differences between each $\lambda$ state for the Lennard-Jones potential gives the non-electrostatic contribution $\Delta G_{\text {nele }}$ to the hydration free energy and the Coulomb 
potential gives the electrostatic contribution $\Delta G_{\text {ele }}$. By adding these two contributions, nonelectrostatic and electrostatic, it is obtained the total ionic hydration free energy, $G^{h y d}=$ $\Delta G_{\text {nele }}+\Delta G_{\text {ele }}$.

The temperature dependence of hydration free energy $G_{i}^{\text {hyd }}(T)$ of each ion was investigated for several force field parameters (as described below), and thirteen temperatures ranging between 293 and $353 \mathrm{~K}$ temperatures in the range of $5 \mathrm{~K}$. From the linear best fitting of $G_{i}^{\text {hyd }}(T)$ for each ion, we obtained the hydration entropy, i.e. the rate of change of $G_{i}^{\text {hyd }}$ with temperature, $S_{i}^{\text {hyd }}=-d G_{i}^{\text {hyd }} / d T$. These results were used to calculate ionic thermodiffusive properties. In this context, we also tested the proportionality factor proposed by Takeyama and Nakashima ${ }^{31}$ between the single-ion heat of transport and the ionic hydration entropy in water, $Q_{i}^{*}(\mathrm{MD} / \mathrm{TN})=-f_{i}^{T N} T S_{i}^{\text {hyd }}(T)$ where the scaling factor $f_{i}^{T N}$ is 0.348 for $\mathrm{Cl}^{-}, 0.383$ for $\mathrm{OH}^{-}, 0.439$ for $\mathrm{Na}^{+}, 0.450$ for $\mathrm{H}_{3} \mathrm{O}^{+}, 0.457$ for $\mathrm{K}^{+}$, and 0.555 for $T M A^{+}$and $T B A^{+}$.

Self diffusion coefficients $D_{i}$ were calculated using Einstein relation between the mean square displacement (MSD) ${ }^{71}$ of the ion and its self diffusion coefficient $D_{i}$ using the $g m x$ msd command available in the GROMACS package. We obtained the MSD of the ion center of mass at five temperatures $(293,298,313,333,353 \mathrm{~K})$ in the equilibrium stage of cMD simulations and using 50 thousand configurations separated by $200 \mathrm{fs}$ in a trajectory of 10 $n s$. By the linear best fitting of the MSD curve in its linear regime, around 0 and $3 n s$, we calculated the ion diffusion coefficient $D_{i}$.

The force field parameters adopted in the MD simulations were: for water the SPC/E model $^{72}$ and TIP3P model ${ }^{73}$ and the traditional models available in literature for $\mathrm{Na}^{+}, \mathrm{K}^{+}$ and $\mathrm{Cl}^{-}$, which we will be referred to as: Aqvist, ${ }^{48}$ Dang, ${ }^{45,49}$ Jorgensen, ${ }^{46}$ Roux, ${ }^{47,50}$ and Canongia $^{44}$ (the latter was used only for $\mathrm{Cl}^{-}$). For $T M A^{+}$and $T B A^{+}$, we adopted the OPLS-AA force field ${ }^{74,75}$ for Lennard-Jones (LJ) potential parameters $(\varepsilon$ and $\sigma$ for $C, H$ and $N$ ) and bonded potential parameters (bond distances, bonds angles and dihedral angles); for Coulomb potential, atomic charges were calculated using the CHELPG procedure ${ }^{76}$ to 
fit the electrostatic potential calculated with quantum mechanics (QM) in an optimized geometry of the ions, including the solvent polarization with the Polarized Continuum model (PCM). ${ }^{77}$ The QM calculations were performed using the MP2 theory ${ }^{78}$ and the basis set cc-pVDZ. ${ }^{79}$ This procedure has been applied successfully in previous works. ${ }^{80-82}$ For $\mathrm{H}_{3} \mathrm{O}^{-}$, we employed the model proposed by Netz. ${ }^{51}$ For $\mathrm{OH}^{-}$, we employed the models: Netz, ${ }^{51} 20$ sites ${ }^{52}$ and Jorgensen. ${ }^{74}$ Furthermore, for $\mathrm{H}_{3} \mathrm{O}^{-}$and $\mathrm{OH}^{-}$, we also used a new set of force

field parameters proposed by us to better describe their hydration free energies at room temperature. For both ions, we obtained their QM optimized geometries at MP2/aug-ccpVQZ ${ }^{79}$ level and the CHELPG atomic charges (only for hydroxide) at MP4(SDQ) ${ }^{83} /$ aug- $^{-}$ cc-pVQZ level in water solution with solvent polarization included with the PCM model. For $O H^{-}$, we adopted the LJ parameters $\left(\varepsilon\right.$ and $\sigma$ ) of the UFF force field, ${ }^{84}$ but by rescaling the $\sigma$ value of Oxygen by 1.3. For $\mathrm{H}_{3} \mathrm{O}^{-}$, we adopted the LJ plus Coulomb parameters proposed by Netz, but rescaling the $\sigma$ value of oxygen by 0.95 . These rescaling factors were obtained after testing several values and they were selected as the best option to better describe hydration free energy of $\mathrm{OH}^{-}$and $\mathrm{H}_{3} \mathrm{O}^{-}$at room temperature. Thus, in this work, we proposed the following set of force field parameters: for $\mathrm{OH}^{-}\left(q_{O}=-1.28, q_{H}=0.28, \varepsilon_{O}=0.251 \mathrm{~kJ} / \mathrm{mol}\right.$, $\left.\varepsilon_{H}=0.184 \mathrm{~kJ} / \mathrm{mol}, \sigma_{O}=0.405 \mathrm{~nm}, \sigma_{H}=0.257 \mathrm{~nm}\right)$ and for $\mathrm{H}_{3} \mathrm{O}^{-}\left(q_{O}=-1.4, q_{H}=0.8\right.$, $\left.\varepsilon_{O}=0.8 \mathrm{~kJ} / \mathrm{mol}, \varepsilon_{H}=0.0 \mathrm{~kJ} / \mathrm{mol}, \sigma_{O}=0.295 \mathrm{~nm}, \sigma_{H}=0.0 \mathrm{~nm}\right)$. For both $O H^{-}$and $\mathrm{H}_{3} \mathrm{O}^{-}$, we adopted the $\mathrm{UFF}^{84}$ bonded parameters. All QM calculations were performed using Gaussian 09 package. ${ }^{85}$ All information about the geometry of polyatomic ions and force field parameters for all ions used in the simulations are available in SI.

\section{Acknowledgement}

The authors thank the National Institute of Science and Technology of Complex Fluids (INCT-FCx) for the CNPq grant 141260/2017-3 and FAPESP grant 2014/50983-3; CAPES concerning the BioMol project 23038.004630/2014-35; FAPESP grants 2017/11631-2, 2019/10433- 
8 and 2016/24531-3; High Performance Computing of USP (HPC-USP) for computational resources.

\section{Supporting Information Available}

More information concerning the force field parameters, radial distribution functions, hydration free energy terms, single-ion heat of transport, reduced single-ion mass current, effective mass diffusion, thermodiffusion, salt Soret and Seebeck coefficients are given in the SI.

\section{References}

(1) Zhao, D.; Wang, H.; Khan, Z. U.; Chen, J. C.; Gabrielsson, R.; Jonsson, M. P.; Berggren, M.; Crispin, X. Ionic thermoelectric supercapacitors. Energy Environment Science 2016, 9, 1450-1457.

(2) Li, T.; Zhang, X.; Lacey, S. D.; Mi, R.; Zhao, X.; Jiang, F.; Song, J.; Liu, Z.; Chen, G.; Dai, J.; Yao, Y.; Das, S.; Yang, R.; Briber, R. M.; Hu, L. Cellulose ionic conductors with high differential thermal voltage for low-grade heat harvesting. Nature Materials 2019 ,

(3) Bonetti, M.; Nakamae, S.; Roger, M.; Guenoun, P. Huge seebeck coefficient in nonaqueous electrolytes. Journal of Chemical Physics 2011, 134, 114513.

(4) Kim, H. S.; Liu, W.; Chen, G.; Chu, C.-W.; Ren, Z. Relationship between thermoelectric figure of merit and energy conversion efficiency. Proceedings of the National Academy of Sciences 2015, 112, 8205-8210.

(5) Bonetti, M.; Nakamae, S.; Huang, B. T.; Salez, T. J.; Wiertel-Gasquet, C.; Roger, M. Thermoelectric energy recovery at ionic-liquid/electrode interface. The Journal of Chemical Physics 2015, 142, 244708. 
(6) Khler, W.; Morozov, K. I. The Soret Effect in Liquid Mixtures A Review. J. NonEquilib. Thermodyn. 2016, 41, 151-197.

(7) Wurger, A. Thermal non-equilibrium transport in colloids. Reports on Progress in Physics 2010, 73, 126601.

(8) Agar, J. N.; Mou, C. Y.; Lin, J. L. Single-ion heat of transport in electrolyte solutions: a hydrodynamic theory. J. Phys. Chem 1989, 93, 2079-2082.

(9) Zhao, D.; Martinelli, A.; Willfahrt, A.; Fischer, T.; Bernin, D.; Khan, Z. U.; Shahi, M.; Brill, J.; Jonsson, M. P.; Fabiano, S.; Crispin, X. Polymer gels with tunable ionic Seebeck coefficient for ultra-sensitive printed thermopiles. Nature Communications 2019, 10, 1093.

(10) Ludwig, C. Diffusion zwischen ungleich erwrmten orten gleich zusammengesetzter lsungen. Sitzungsber. Adad. Wiss. Wien 1856, 20, 539.

(11) Soret, C. Arch. Sci. Phys. Nat. Geneva 1879, 2, 48.

(12) Eastman, E. D. THEORY OF THE SORET EFFECT. Journal of the American Chemical Society 1928, 50, 283-291.

(13) Eastman, E. D. The Thermodynamics of Non-Isothermal Systems. Journal of the American Chemical Society 1927, 49, 794-795.

(14) Agar, J. N.; Turner, J. C. R. Thermal Diffusion in Solutions of Electrolytes. Proceedings of the Royal Society of London A 1960, 255, 307-330.

(15) Agar, J. N. Thermogalvanic Cells, in Advances in Electrochemistry and Electrochemical Engineering, pp. 31-121; Advances in Electrochemistry and Electrochemical Engineering, pp. 31-121; Interscience New York, 1963. 
(16) Römer, F.; Wang, Z.; Wiegand, S.; Bresme, F. Alkali Halide Solutions under Thermal Gradients: Soret Coefficients and Heat Transfer Mechanisms. The Journal of Physical Chemistry B 2013, 117, 8209-8222.

(17) Eslahian, K. A.; Maskos, M. Hofmeister effect in thermal field-flow fractionation of colloidal aqueous dispersions. Colloids and Surfaces A: Physicochemical and Engineering Aspects 2012, 413, $65-70$.

(18) Eslahian, K. A.; Majee, A.; Maskos, M.; Wurger, A. Specific salt effects on thermophoresis of charged colloids. Soft Matter 2014, 10, 1931 - 1936.

(19) Niether, D.; Kawaguchi, T.; Hovancová, J.; Eguchi, K.; Dhont, J. K. G.; Kita, R.; Wiegand, S. Role of Hydrogen Bonding of Cyclodextrin-Drug Complexes Probed by Thermodiffusion. Langmuir 2017, 33, 8483-8492.

(20) Niether, D.; Kriegs, H.; Dhont, J. K. G.; Wiegand, S. Peptide model systems: Correlation between thermophilicity and hydrophobicity. The Journal of Chemical Physics 2018, $149,044506$.

(21) Stout, R. F.; Khair, A. S. Diffuse charge dynamics in ionic thermoelectrochemical systems. Physical Review E 2017, 96, 022604.

(22) Janssen, M.; Bier, M. Transient response of an electrolyte to a thermal quench. Physical Review E 2019, 99, 042136.

(23) Reichl, M.; Herzog, M.; Gatz, A.; Braun, D. Why Charged Molecules Move Across a Temperature Gradient: The Role of Electric Fields. Physical Review Letter 2014, 112, 198101.

(24) Sehnem, A. L.; Figueiredo Neto, A. M.; Aquino, R.; Campos, A. F. C.; Tourinho, F. A.; Depeyrot, J. Temperature dependence of the Soret coefficient of ionic colloids. Physical Review E 2015, 92, 042311. 
(25) Sehnem, A. L.; Neto, A. M. F.; Niether, D.; Wiegand, S. Diffusiophoresis as Ruling Effect: Influence of Organic Salts on Thermodiffusion of Iron Oxide Nanoparticles. Physical Review E 2018, 98, 062615.

(26) Huang, B. T.; Roger, M.; Bonetti, M.; Salez, T. J.; Wiertel-Gasquet, C.; Dubois, E.; Gomes, R. C.; Demouchy, G.; Mariguet, G.; Peyre, V.; Kouyata, M.; Filomeno, C. L.; Depeyrot, J.; Tourinho, F. A.; Perzynski, R.; Nakamae, S. Thermoelectricity and thermodiffusion in charged colloids. Journal of Chemical Physics 2015, 143, 054902.

(27) Salez, T. J.; Huang, B. T.; Rietjens, M.; Bonetti, M.; Wiertel-Gasquet, C.; Roger, M.; Filomeno, C. L.; Dubois, E.; Perzynski, R.; Nakamae, S. Can charged colloidal particles increase the thermoelectric energy conversion efficiency? Physical Chemistry Chemical Physics 2017, 19, 9409-9416.

(28) Duhr, S.; Braun, D. Why molecules move along a temperature gradient. Proceedings of the National Academy of Sciences 2006, 103, 19678 - 19682.

(29) Astumian, R. D. Coupled transport at the nanoscale: The unreasonable effectiveness of equilibrium theory. Proceedings of the National Academy of Sciences 2007, 104, 3-4.

(30) Würger, A. Is Soret equilibrium or non-equilibrium effect? Comptes Rendus Mecanique 2013, 341, 438 - 448, 10th International Meeting on Thermodiffusion.

(31) Takeyama, N.; Nakashima, K. Proportionality of Intrinsic Heat of Transport to Standard Entropy of Hydration for Aqueous Ions. Journal of Solution Chemistry 1988, 17, 305-325.

(32) Carlsson, J.; Aqvist, J. Absolute Hydration Entropies of Alkali Metal Ions from Molecular Dynamics Simulations. Journal of Physical Chemistry B 2009, 113, 10255 - 10260.

(33) Niether, D.; Kriegs, H.; Dhont, J. K. G.; Wiegand, S. Peptide model systems: Cor- 
relation between thermophilicity and hydrophilicity. The Journal of Chemical Physics 2018, 149, 044506.

(34) Niether, D.; Lecce, S. D.; Bresme, F.; Wiegand, S. Unraveling the hydrophobicity of urea in water using thermodiffusion: implications for protein denaturation. Physical Chemistry Chemical Physics 2018, 20, 1012 - 1020.

(35) Lecce, S. D.; Albrecht, T.; Bresme, F. A computational approach to calculate the heat of transport of aqueous solutions. Scientific Reports 2017, 7, 44833.

(36) Lecce, S. D.; Bresme, F. Soret coefficients and thermal conductivities of alkali halide aqueous solutions via non-equilibrium molecular dynamics simulations. Molecular Simulation 2019, 45, 351-357.

(37) Astumian, R. D. The unreasonable effectiveness of equilibrium theory for interpreting nonequilibrium experiments. American Journal of Physics 2006, 74, 683-688.

(38) Schmid, R.; Miah, A. M.; Sapunov, V. N. A new table of the thermodynamic quantities of ionic hydration: values and some applications (enthalpy-entropy compensation and Born radii). Physical Chemistry Chemical Physics 2000, 2, 97-102.

(39) Marcus, Y. A simple empirical model describing the thermodynamics of hydration of ions of widely varying charges, sizes, and shapes. Biophysical chemistry 1994, 51, 111127.

(40) Pliego Jr, J. R.; Riveros, J. M. New values for the absolute solvation free energy of univalent ions in aqueous solution. Chemical Physics Letters 2000, 332, 597-602.

(41) Light, T. S.; Licht, S.; Bevilacqua, A. C.; Morash, K. R. The fundamental conductivity and resistivity of water. Electrochemical and Solid State Letters 2004, 8, E16.

(42) Sehnem, A. L.; Niether, D.; Wiegand, S.; Figueiredo Neto, A. M. Thermodiffusion in 
monovalent organic salts in water. The Journal of Physical Chemistry B 2018, 122, 4093-4100.

(43) Sehnem, A. L.; Neto, A. M. F. Dynamic response of a thermoelectric cell induced by ion thermodiffusion. arXiv:1911.11799 2019,

(44) Canongia Lopes, J. N.; Deschamps, J.; Pádua, A. A. Modeling ionic liquids using a systematic all-atom force field. The Journal of Physical Chemistry B 2004, 108, 20382047.

(45) Smith, D. E.; Dang, L. X. Computer simulations of $\mathrm{NaCl}$ association in polarizable water. The Journal of Chemical Physics 1994, 100, 3757-3766.

(46) Jensen, K. P.; Jorgensen, W. L. Halide, ammonium, and alkali metal ion parameters for modeling aqueous solutions. Journal of Chemical Theory and Computation 2006, 2, 1499-1509.

(47) Roux, B. Valence selectivity of the gramicidin channel: a molecular dynamics free energy perturbation study. Biophysical journal 1996, 71, 3177-3185.

(48) Aqvist, J. Ion-water interaction potentials derived from free energy perturbation simulations. The Journal of Physical Chemistry 1990, 94, 8021-8024.

(49) Dang, L. X.; Kollman, P. A. Free energy of association of the K+: 18-crown-6 complex in water: a new molecular dynamics study. The Journal of Physical Chemistry 1995, $99,55-58$.

(50) Beglov, D.; Roux, B. Finite representation of an infinite bulk system: solvent boundary potential for computer simulations. The Journal of chemical physics 1994, 100, 90509063.

(51) Bonthuis, D. J.; Mamatkulov, S. I.; Netz, R. R. Optimization of classical nonpolarizable force fields for $\mathrm{OH}-$ and $\mathrm{H} 3 \mathrm{O}+$. The Journal of Chemical Physics 2016, 144, 104503. 
(52) Ufimtsev, I. S.; Kalinichev, A. G.; Martinez, T. J.; Kirkpatrick, R. J. A charged ring model for classical OH-(aq) simulations. Chemical Physics Letters 2007, 442, 128-133.

(53) Berg, H. Random walks in Biology; Princeton University Press, 1983.

(54) Day, T. J.; Soudackov, A. V.; Čuma, M.; Schmitt, U. W.; Voth, G. A. A second generation multistate empirical valence bond model for proton transport in aqueous systems. The Journal of Chemical Physics 2002, 117, 5839-5849.

(55) Lee, S. H.; Rasaiah, J. C. Proton transfer and the mobilities of the $\mathrm{H}+$ and $\mathrm{OH}-$ ions from studies of a dissociating model for water. The Journal of Chemical Physics 2011, 135,124505 .

(56) Ojamäe, L.; Shavitt, I.; Singer, S. J. Potential models for simulations of the solvated proton in water. The Journal of chemical physics 1998, 109, 5547-5564.

(57) Bringuier, E. On the notion of thermophoretic velocity. Philosophical Magazine 2007, $87,873-883$.

(58) De Groot, S. Sur la thermodynamique de quelques processus irréversibles. II. Diffusion thermique et phénomènes connexes. J. Phys. Radium 1947, 8, 193-200.

(59) Sehnem, A. L.; Janssen, M. Determining single-ion Soret coefficients from the transient electrolyte Seebeck effect. https://arxiv.org/pdf/2006.11081 2020,

(60) Wang, H.; Zhao, D.; Khan, Z. U.; Puzinas, S.; Jonsson, M. P.; Berggren, M.; Crispin, X. Ionic Thermoelectric Figure of Merit for Charging of Supercapacitors. Advanced Electronic Materials 2017, 3, 1700013.

(61) Van Der Spoel, D.; Lindahl, E.; Hess, B.; Groenhof, G.; Mark, A. E.; Berendsen, H. J. GROMACS: fast, flexible, and free. Journal of computational chemistry 2005, 26, $1701-1718$. 
(62) Wu, X.; Brooks, B. R. Self-guided Langevin dynamics simulation method. Chemical Physics Letters 2003, 381, 512-518.

(63) Bussi, G.; Donadio, D.; Parrinello, M. Canonical sampling through velocity rescaling. The Journal of chemical physics 2007, 126, 014101.

(64) Berendsen, H. J.; Postma, J. v.; van Gunsteren, W. F.; DiNola, A.; Haak, J. R. Molecular dynamics with coupling to an external bath. The Journal of chemical physics $\mathbf{1 9 8 4 ,}$ 81, 3684-3690.

(65) Essmann, U.; Perera, L.; Berkowitz, M. L.; Darden, T.; Lee, H.; Pedersen, L. G. A smooth particle mesh Ewald method. The Journal of chemical physics 1995, 103, 85778593.

(66) Hockney, R. W.; Goel, S.; Eastwood, J. W. Quiet high-resolution computer models of a plasma. Journal of Computational Physics 1974, 14, 148 - 158.

(67) Van Gunsteren, W. F.; Berendsen, H. J. A leap-frog algorithm for stochastic dynamics. Molecular Simulation 1988, 1, 173-185.

(68) Goga, N.; Rzepiela, A.; De Vries, A.; Marrink, S.; Berendsen, H. Efficient algorithms for Langevin and DPD dynamics. Journal of chemical theory and computation 2012, 8, 3637-3649.

(69) Hess, B.; Bekker, H.; Berendsen, H. J. C.; Fraaije, J. G. E. M. LINCS: a linear constraint solver for molecular simulations. Journal of Computational Chemistry 1997, 18, 14631472.

(70) Bennett, C. H. Efficient estimation of free energy differences from Monte Carlo data. Journal of Computational Physics 1976, 22, 245-268.

(71) Frenkel, D.; Smit, B. Understanding Molecular Simulation Academic Press. San Diego 1996, 27-33. 
(72) Berendsen, H. HJC Berendsen, JR Grigera, and TP Straatsma, J. Phys. Chem. 91, 6269 (1987). Journal of Physical Chemistry 1987, 91, 6269.

(73) Jorgensen, W. L.; Chandrasekhar, J.; Madura, J. D.; Impey, R. W.; Klein, M. L. Comparison of simple potential functions for simulating liquid water. The Journal of chemical physics 1983, 79, 926-935.

(74) Jorgensen, W. L.; Maxwell, D. S.; Tirado-Rives, J. Development and testing of the OPLS all-atom force field on conformational energetics and properties of organic liquids. Journal of the American Chemical Society 1996, 118, 11225-11236.

(75) Kaminski, G. A.; Friesner, R. A.; Tirado-Rives, J.; Jorgensen, W. L. Evaluation and reparametrization of the OPLS-AA force field for proteins via comparison with accurate quantum chemical calculations on peptides. The Journal of Physical Chemistry B 2001, $105,6474-6487$.

(76) Breneman, C. M.; Wiberg, K. B. Determining atom-centered monopoles from molecular electrostatic potentials. The need for high sampling density in formamide conformational analysis. Journal of Computational Chemistry 1990, 11, 361-373.

(77) Scalmani, G.; Frisch, M. J. Continuous surface charge polarizable continuum models of solvation. I. General formalism. The Journal of chemical physics 2010, 132, 114110.

(78) Head-Gordon, M.; Pople, J. A.; Frisch, M. J. MP2 energy evaluation by direct methods. Chemical Physics Letters 1988, 153, 503-506.

(79) Dunning Jr, T. H. Gaussian basis sets for use in correlated molecular calculations. I. The atoms boron through neon and hydrogen. The Journal of Chemical Physics 1989, 90, 1007-1023.

(80) Franco, L. R.; Brandão, I.; Fonseca, T. L.; Georg, H. C. Elucidating the structure of 
merocyanine dyes with the ASEC-FEG method. Phenol blue in solution. The Journal of chemical physics 2016, 145, 194301.

(81) Brandão, I.; Franco, L. R.; Fonseca, T. L.; Castro, M. A.; Georg, H. C. Confirming the relationship between first hyperpolarizability and the bond length alternation coordinate for merocyanine dyes. The Journal of Chemical Physics 2017, 146, 224505.

(82) Cezar, H. M.; Canuto, S.; Coutinho, K. Solvent effect on the syn/anti conformational stability: A comparison between conformational bias Monte Carlo and molecular dynamics methods. International Journal of Quantum Chemistry 2019, 119, e25688.

(83) Krishnan, R.; Pople, J. A. Approximate fourth-order perturbation theory of the electron correlation energy. International Journal of Quantum Chemistry 1978, 14, 91-100.

(84) Garberoglio, G. OBGMX: A web-based generator of GROMACS topologies for molecular and periodic systems using the universal force field. Journal of computational chemistry 2012, 33, 2204-2208.

(85) Frisch, M. J.; Trucks, G. W.; Schlegel, H. B.; Scuseria, G. E.; Robb, M. A.; Cheeseman, J. R.; Scalmani, G.; Barone, V.; Mennucci, B.; Petersson, G. A.; Nakatsuji, H.; Caricato, M.; Li, X.; Hratchian, H. P.; Izmaylov, A. F.; Bloino, J.; Zheng, G.; Sonnenberg, J. L.; Hada, M.; Ehara, M.; Toyota, K.; Fukuda, R.; Hasegawa, J.; Ishida, M.; Nakajima, T.; Honda, Y.; Kitao, O.; Nakai, H.; Vreven, T.; Montgomery, J. A.; Jr.,; Peralta, J. E.; Ogliaro, F.; Bearpark, M.; Heyd, J. J.; Brothers, E.; Kudin, K. N.; Staroverov, V. N.; Kobayashi, R.; Normand, J.; Raghavachari, K.; Rendell, A.; Burant, J. C.; Iyengar, S. S.; Tomasi, J.; Cossi, M.; Rega, N.; Millam, J. M.; Klene, M.; Knox, J. E.; Cross, J. B.; Bakken, V.; Adamo, C.; Jaramillo, J.; Gomperts, R.; Stratmann, R. E.; Yazyev, O.; Austin, A. J.; Cammi, R.; Pomelli, C.; Ochterski, J. W.; Martin, R. L.; Morokuma, K.; Zakrzewski, V. G.; Voth, G. A.; Salvador, P.; Dannen- 
berg, J. J.; Dapprich, S.; Daniels, A. D.; Farkas, O.; Foresman, J. B.; Ortiz, J. V.;

Cioslowski, J.; Fox, D. J. Gaussian 09, Revision D.01; Inc., Wallingford CT, 2013.

\section{For Table of Contents Only}

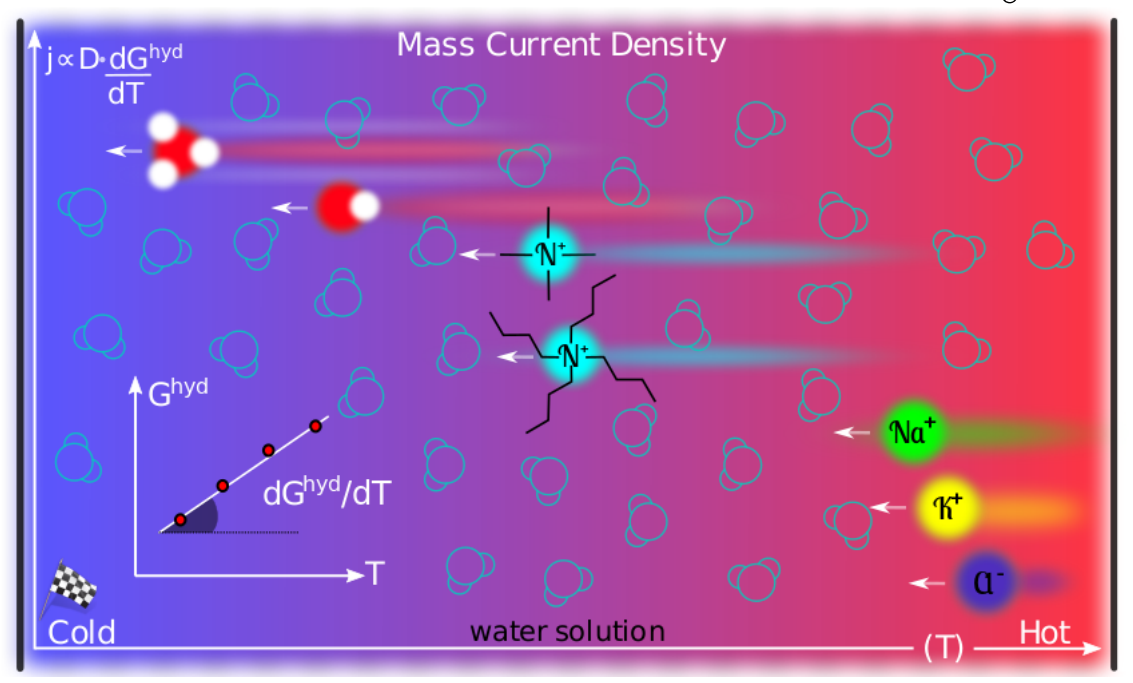

